Editorial

\title{
Acknowledgment to Reviewers of Processes in 2020
}

\section{Processes Editorial Office}

Citation: Processes Editorial Office. Acknowledgment to Reviewers of Processes in 2020. Processes 2021, 9, 211. https://doi.org/10.3390/pr902 0211

Published: 23 January 2021

Publisher's Note: MDPI stays neutral with regard to jurisdictional claims in published maps and institutional affiliations.

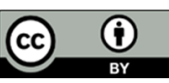

Copyright: (c) 2021 by the author. Licensee MDPI, Basel, Switzerland. This article is an open access article distributed under the terms and conditions of the Creative Commons Attribution (CC BY) license (http://creativecommons.org/licenses /by/4.0/).

MDPI AG, St. Alban-Anlage 66, 4052 Basel, Switzerland

Peer review is the driving force of journal development, and reviewers are gatekeepers who ensure that Processes maintains its standards for the high quality of its published papers. Thanks to the cooperation of our reviewers, in 2020, the median time to first decision was 14 days and the median time to publication was 35 days. The editors would like to express their sincere gratitude to the following reviewers for their precious time and dedication, regardless of whether the papers were finally published:

A.T. Borojeni, Azadeh

Abate, Giada

Abba, Mohammed L.

Abdallah, Shaaban

Abdelouahed, Lokmane

Abdullayev, Amanmyrat

Abejón, Ricardo

Abella, Pura Alfonso

Abghari, Ali

Aboudi, Jacob

Abruzzo, Angela

Abu-Niaaj, Lubna

Acedo, Jeella Z.

Acedos, Miguel G.

Acharya, Bishnu

Acho, Leonardo

Achuthan, Ajit

Ačkar, Đurđica

Acosta Contreras, Florentina Niuris

Acree Jr., William E.

Adamczyk, Marcin

Adamenko, Kinga

Adamus, Grazyna

Adekunle, Adeshina

Adeniyi, Akinola

Adera, Solomon

Adhikari, Rajendra Singh

Adhikari, Ram C

Adiletta, Giuseppina

Adjé, Félix

Adriana, Tokar

Afa Michael, Inikuro

Afify, Ahmed Sabry

Afonso, Armando Silva

Afshari, Khashayar
Agar, Ertan

Agatemor, Christian

Aggas, John

Agili, Sedig S.

Agnieszka, Szopa

Agrosi, Giovanna

Ahamed, Md Shamim

Ahluwalia, Pankaj

Ahmad, Hafiz

Ahmadi, Mojtaba

Ahmadvand, Seyedsaeid

Ahmed Mohamed, Mohamed Hassan

Ahmed, Abdullahi

Ahmed, Hesham M.

Ahmed, Sheikh

Ahn, Sang-hyun

Aidoo, Anthony

Aimi, Junko

Aivazidou, Eirini

Akafuah, Nelson K.

Akbar, Muhammad K.

Akbari Garakani, Mohammad

Akingbade, Katherine L.

Akkermans, Rinie

Alaluss, Khaled

Alar, Vesna

Alberti, Marcos G.

Alberti, Tommaso

Alborz, Niknam

Albrektienè, Ramunè

Alcantara, Andres

Alcántara-Ávila, Jesús Rafael

Alcaraz, Miguel

Alejski, Krzysztof

Alessandro, Dell'Era 
Alessio, Dessì

Alexandri, Alexandra

Alexandri, Maria

Alexovič, Michal

Al-Fatimi, Mohamed

Alfredo, Katherine

Algayyim, Sattar

Alhasheem, Mohammed

Ali, Babkir

Ali, Naser

Ali, Samim

Aliaño González, María José

Alijagić, Jasminka

Alina, Brzeczek-Szafran

Alinovi, Marcello

Alique Amor, David

Aljohani, Tawfiq

Alkan, Bugra

Alkasrawi, Malek

Allais, Florent

Allazadeh, Mohammad

Allen, Norman S.

Allgower, Frank

Almadhor, Ahmad

Almásy, László

Almeida, Maria Judite

Alsaaty, Falih

Alsajri, Firas Ahmed

Al-Shakry, Badar

Al-Shibaany, Zeyad

Alsultan, Mohammed

Altraja, Alan

Alugubelly, Navatha

Alvarez, Juan B.

Alvarez, Mar

Álvarez, María S.

Álvarez, Paloma

Alvarez-Malmagro, Julia

Alves Miller, Fátima

Alves, Ana Catarina

Alves, Joana

Alves, Luis G.

Alves-Silva, Jorge M.

Alwaeli, Mohamed

Amadio, Emanuele

Amani, Ahmad

Amanowicz, Łukasz

Amaro, Ana

Ambrose, Stephen

Amer, Yousef

Ameur, Khaled
Amigo, José-Manuel

Amin, Farhan

Amirkhosravi, Mehrad

Ammendola, Paola

Amro, Moh'd M.

Amundarain Irizar, Aiert

An, Chunjiang

Anagnostis, Athanasios

Anajafi, Hamidreza

Anand, Prashant

Ananthakrishnan, Soundaram Jeevarathinam

Anastassova, Neda

Ancellin, Matthieu

Andaluri, Gangadhar

Anderson, Danial

Andersson, Anton

Andersson, Klas

Andiappan, Viknesh

Andraka, Dariusz

Andrés, Eduardo

Andresen Eguiluz, Roberto

Andresini, Michael

Andriolo, Jessica

Andrychowicz, Mateusz

Andrzejewski, Jacek

Angelini, Gino

Angeloni, Simone

Anggraini, Vivi

Anibal, Jaime

Annesini, Maria Cristina

Ansari, Amir

Ansari, Sardar

Ansell, Troy Y.

Anselmet, Fabien

Anthony, Edward

Antolak, Hubert

Antolini, Ermete

Antoniewska, Agata

Antonopoulou, Georgia

Antonov, Alexander S.

Antunes, Dulce

Antunes, Francisco

Anugwom, Ikenna

Anyaiwe, Destiny E. O.

Anyszka, Rafał

Aphale, Ashish

Apostolidis, Panos

Arabzadeh, Vahid

Aramendia, Iñigo

Aranaz, Paula

Aranda-Escolástico, Ernesto 
Arato, Elisabetta

Araújo, João M. M.

Araujo, Maria E. M.

Arena, Antonella

Arena, Katia

Ares-Pernas, Ana

Argyropoulos, Christos

Arias-Estévez, Manuel

Arifuzzaman, Mohammad

Aristia, Gabriela

Arnaboldi, Serena

Arnold, Ulrich

Aronica, Laura Antonella

Arora, Neha

Arrabal-Campos, Francisco Manuel

Arranz, Miguel Castaño

Arregi, Aitor

Arrigo, Rossella

Arsiccio, Andrea

Asamoah, Richmond

Aschilean, Ioan

Ascrizzi, Roberta

Ashour, Mohamed Lotfy

Askari, Omid

Aslani, Mohamad

Assaf, Khaleel Ibrahim

Assirelli, Alberto

Astorino, Annabella

Asvapathanagul, Pitiporn

Atanasov, Vasil

Atanassova, Maria

Atia, Hanan

Atkins, Martin

Atla, Veerendra

Atta-Obeng, Emmanuel

Augustyniak, Adrian

Auh, Joong-Hyuck

Aung, Kendrick

Avdicevic, Maja Zebic

Avgouropoulos, George

Awolayo, Adedapo

Axelevitch, Alexander

Ayankojo, 4Akinrinade George

Ayuso, José María

Azad, Madhar

Azar, Ahmad Taher

Azis, Konstantinos

Azrague, Kamal

Baaliña Insua, Álvaro

Babič, František

Bacanin, Nebojsa
Baccoli, Roberto

Badawi, Michael

Bade, Mehar

Badea, Mihaela

Badr, Sara

Bae, Hyerim

Bae, Hyeun-Jong

Baeghbali, Vahid

Baek, Jong-Suep

Baek, Nakhoon

Baena, Francisco Manuel

Baghdadi, Fazia

Baharum, Aslina

Bahi, Addie

Bahmanpouri, Farhad

Bai, Attila

Bai, Lei

Baig, Khurram Shahzad

Bains, William

Baiocco, Silvia

Bak, Andrzej

Bakhshian, Sahar

Bakonyi, Péter

Balacco, Gabriella

Balasingam, Suresh Kannan

Balawejder, Maciej

Baldassari, Sara

Baldi, Franco

Baldini, Mario

Baldino, Lucia

Baldry, Mark

Balduzzi, Francesco

Balestrieri, Eulalia

Baležentis, Tomas

Ballard, Nicholas

Banach, Artur

Banaś, Michał

Banaszkiewicz, Tomasz

Banchelli, Martina

Bandapalli, Obul Reddy

Bandiera, Lucia

Bandini, Serena

Banerjee, Amborish

Banerjee, Deepanwita

Barabanova, Anna I.

Barabasz, Wieslaw

Baranowska, Hanna

Baranyai, László

Barasinski, Anais

Barati, Ziba

Barba, Francisco J. 
Barbera, Orazio

Barbeş, Lucica

Barczak, Agnieszka

Bardi, Alessandra

Barloková, Danka

Barna, Martin

Barnett, Chris J.

Barolo, Massimiliano

Barral Silva, María Teresa

Barreto, Maria

Barrette, Julie

Barsi, Dario

Bartlett, John

Bartocci, Pietro

Bartoli, Mattia

Bartosz, Grzegorz

Bartusch, Hauke

Bartyś, Michał

Barukčić, Marinko

Barwiolek, Magdalena

Basařová, Pavlína

Basen, Mirko

Basile, Teodora

Basse, Nils Tångefjord

Basso, Gianluigi Lo

Bastrzyk, Anna

Batarseh, Feras A.

Batrancea, Larisa

Batterham, Robin J.

Batteux, Michel

Bauer, Christian

Bauer, Roman

Bazan, Patrycja

Bazooyar, Bahamin

Bazylko, Agnieszka

Bazzarelli, Fabio

Beane, Gary

Bearup, Daniel

Beaudoin, Anthony

Bębenek, Ewa

Bedla, Dawid

Bednar, Slavomir

Bedon, Chiara

Bedrov, Dmitry A.

Behrendt, Matthias

Beju, Livia Dana

Bekmukhamedov, Giyjaz E.

Belardelli, Filippo

Belgiorno, Giacomo

Bell, John

Bellemans, Inge
Bellostas Perezgrueso, Ana José

Belyakov, Sergey

Bembenek, Michał

Bendini, Alessandra

Beneda, Károly

Benedict, Barry

Benim, Ali Cemal

Benítez-Mateos, Ana I.

Beniuga, Razvan

Benkhelifa, Hayat

Benko, Božidar

Benohoud, Meryem

Bera, Gopal

Beretta, Gabriele

Berezowski, Marek

Berger, Rob

Bergonzi, Maria Camilla

Berlowska, Joanna

Berna, Àngel

Bernard, Olivier

Bernardino, Raul J.

Bernasowski, Mikołaj

Bernhauerová, Veronika

Berri, David

BERRI, Pier Carlo

Bertani, Roberta

Berton, Paula

Bertoni, Francesco

Bertsch, Arnaud

Bęś, Agnieszka

Besenhard, Maximilian

Bessmertny, Igor A.

Betekhtin, Alexander

Bettache, Nadir

Bettencourt, Ana

Bettini, Simona

Bezrodnykh, Sergei Igorevich

Bezzo, Fabrizio

Bhandari, Manohar Prasad

Bhardwaj, Vikas

Bhat, Sartaj Ahmad

Bhatia, Suresh

Bhethenabotla, Venkat

Bhutta, Muhammad Usman

Białecki, Ryszard

Białobrzeska, Beata

Biancalana, Valerio

BIANCHI, Biagio

Bianco, Giuliana

Biel, Wioletta

Biernat, Jan 
Bierzuńska, Paulina

Bigatao Souza, Joao Luis

Bigda, Rafał

Bila, Alberto

Bilewicz, Aleksander

Bini, Marcella

Biniak-Pieróg, Małgorzata

Binotti, Marco

Birlutiu, Adriana

Biron, David G.

Bis, Marta

Bisbey, Ryan

Bishop, Chris

Bizon, Katarzyna

Bizyukov, Pavel

Blair, Ewen O.

Blanco, Angeles

Blau, Werner

Błażewska, Katarzyna M.

Blecich, Paolo

Block, Stephan

Blondeau, Julien

Blood, Daniel

Blumenfeld Olivares, Javier Andres

Blumer-Schuette, Sara E.

Blunt, Warren

Bo, Song

Bo, Zhenyu

Bobadilla, Luis F.

Bobis, Otilia

Boccardo, Gianluca

Bocko, Jozef

Bode, Christoph

Bodnár, Tomáš

Boer, Dieter

Boesel, Luciano

Boffa, Luisa

Boffito, Daria

Bogacki, Jan

Bogataj, Marija

Bogdanowicz, Krzysztof Artur

Bohdalová, Mária

Bohm, Sivasambu

Bolla, Pradeep Kumar

Bombac, Andrej

Bombek, Gorazd

Bond, Ryan

Bondareva, Nadezhda S.

Bonelli, Francesco

Bonifacio, Maria Addolorata

Bonvin, Dominique
Bonyár, Attila

Boo, Yong Chool

Bórawski, Piotr

Borecki, Michal

Borghi, Francesca

Borodianskiy, Konstantin

Borole, Abhijeet

Boros-Lajszner, Edyta

Borovik, Igor

Borovinskaya, Ekaterina S.

Borowicz, Marcin

Borowski, Marek

Borowski, Piotr

Borra, Vamsi

Borrelli, Emma

Bortz, Michael

Borucka, Anna

Boryczko, Krzysztof

Borys, Krzysztof

Borza, Firuta

Boselli, Emanuele

Boshir Ahmed, Mohammad

Boškoski, Pavle

Bošnjaković, Mladen

Bosso, Luciano

Bostan, Roxana

Botta, Bruno

Botubol-Ares, José Manuel

Boudjadar, Jalil

Bournazou, Mariano Nicolas Cruz

Bouropoulos, Nikolaos

Bouyssiere, Brice

Boy, Virginie

Boyaval, Sébastien

Boysen, Reinhard

Bozena, Gajdzik

Bozena, Sera

Bozhko, Yulia Yu.

Bozkurt, Selim

Bracale, Antonio

Brack, Andre

Braga Vieira, Camila Braga

Brameshuber, Mario

Branco, Vasco

Branco-Vieira, Monique

Brandyk, Andrzej

Brás, Eduardo J. S.

Brasinika, Despina

Brassard, Daniel

Bratko, Denis

Bratu, Polidor 
Braun, Burga

Brauss, Daniel

Brégier, Frédérique

Brehm, Martin

Brenišin, Marek

Brenker, Jason

Bressot, Christophe

Bridgwater, Anthony

Brito, Francisco P.

Brito, Moisés

Brito, Paulo

Brodnianska, Zuzana

Brück, Felix

Brückner, Lisa

Bruzzone, Luca

Bryjak, Marek

Bryner, Elliott

Bub, Sascha

Buciakowski, Mariusz

Bück, Andreas

Budaeva, Vera V.

Budnikova, Yulia

Budzak, Simon

Bufalo, Gennaro

Buffière, Pierre

Bufo, Sabino Aurelio

Bui, Duc-Cuong

Buick, James

Bukhari, Syed Basit Ali

Bukhtiyarova, Galina A.

Bulgariu, Laura

Bulic, Neven

Bulko, Branislav

Bunaziv, Ivan

Bunea, Claudiu Ioan

Burca, Genoveva

Burek, Malgorzata

Burg, Ariela

Burnak, Baris

Burns, Jonathan

Bursi, Oreste S.

Bushell, Trevor

Busila, Mariana

Bustamante, Eliseo

Buzatu, Andrei

Buzzi, Fulvio

Bylka, Jedrzej

Cabeza-Lainez, Jose M.

Cabral-Pinto, Marina M. S.

Cabrera, Wellington M.

Cacak-Pietrzak, Grażyna
Cacciola, Francesco

Caciolli, Antonio

Cacopardo, Ludovica

CADET, Frederic

Caesarendra, Wahyu

Caetano, Nídia

Cai, Yuhang

Caignaert, Guy

Calabrò, Francesco

Calado, Cecília R. C.

Calafel, Itxaso

Caldeirinha, Vitor

Calegari, Roberta

Caleja, Cristina

Calin-Adrian, Comes

Calinescu, Ioan

Calín-Sánchez, Ángel

Calixto, Ana Rita

Calokerinos, Antony C.

Calvo, Lourdes

Camaiti, Mara

Camarda, Kyle

Campi, Cristina

Campo, Riccardo

Campone, Luca

Campos-Rebelo, Rogério

Canepa, Edward

Cao, Jianyun

Capacchione, Carmine

Capaldi, Pietro

Capasso, Raffaele

Capatina, Alexandru

Capezza, Antonio

Capozzi, Luigi Carlo

Capozzi, Vittorio

Cappelletti, Giuseppe

Cappellini, Francesca

Caprio, Fabrizio Di

Capuano, Paolo

Carabineiro, Sonia

Caraman, Sergiu

Carapau, Fernando Manuel Lucas

Carbia Carril, José

Carbone, Rosario

Cardona-morales, Oscar

Carere, Carlo

Carfora, Anna

Carleer, Robert

Carles Escola-Gil, Joan

Carli, Raffaele

Carlo, Petrarca 
Carlomagno, Ilaria

Carlson, Ross

Carmo-Calado, Luis

Carmona, Estefanía Núñez

Caron, Rosemary

Carradori, Simone

Carrier, Marion

Carrillo, Celia

Carrubba, Alessandra

Carullo, Gabriele

Caruso, Gabriella

Caruso, Marisa C.

Caruso, Ugo

Carvalho, Lara

Casado, Natalia

Casado-Coterillo, Clara

Casal Fabrega, Joaquim

Casale, Giuseppe Rocco

Casals, Lluc Canals

Cásedas, Guillermo

Casertano, Marcello

Casimiro, Maria Helena

Casisi, Melchiorre

Casoni, Dorina

Cassidy, Daniel P.

Casteleiro-Roca, Jose Luis

Castelletto, Valeria

Castro-Dominguez, Bernardo

Cataldo, Salvatore

Catalina, Mirela

Catena, Francesco

Catizzone, Enrico

Catunescu, Giorgiana

Cavalaglio, Gianluca

Cavas-Martínez, Francisco

Caviglia, Caterina

Cavone, Graziana

Cecchi, Lorenzo

Cecilia, Hodur

Cecilia, Juan Antonio

Cecone, Claudio

Celeiro, María

Čepulienè, Rita

Cerciello, Francesca

Čereška, Audrius

Cerqueira, Laura

Cerrato, Andrea

Cerrone, Federico

Cesário, Rute

Cesmeci, Sevki

Ceyhan, Sercan
Cha, Hyun Gil

Cha, Seung Hyeok

Chaber, Radosław

Chae, Sung Ho

Chai, Lei

Chai, Lilong

Chakraborty, Amlan

Chakraborty, Basabi

Champagne, Victor K.

Chan, Ben C. L.

Chan, Der-Sheng

Chan, Edward

CHAN, JOSHUA

Chan, Leon

Chan, Yen-Hsin

Chanda, Diptiman

Chandrudu, Saranya

Chang, Hai-Chou

Chang, Han

Chang, Jih-Hsing

Chang, Sheng-Po

Chang, Tai-Woo

Chang, Yau-Zen

Chaouch, Mounir

Chapela, Sergio

Charalampopoulos, Loannis

Charmas, Barbara

Charvátová, Hana

Charykov, Nikolay A.

Chatzidoukas, Christos

Chau, Henry

Chaudhury, Anwesha

Chauhan, Neeraj

Chaussonnet, Geoffroy

Chavez, Ferman

Chekurov, Sergei

Chen, Baixin

Chen, Bin-Huei

Chen, Chiachung

Chen, Chihchen

Chen, Dyi-Cheng

Chen, Feng

Chen, George

Chen, Haoyuan

Chen, Hongtian

Chen, Huizhi

Chen, James Ming

Chen, Jem-Kun

Chen, Liang-Yu

Chen, Mingchih

Chen, Pao Chi 
Chen, Qingyu

Chen, Shih-Hsin

Chen, Syuan-Yi

Chen, Tsung-Chia

Chen, Wan-Jiun

Chen, Wei-Hsin

Chen, Wenxi

Chen, Xi

Chen, Xiaorui

Chen, Xin

Chen, Xixian

Chen, Yih-Fung

Chen, Yubin

Chen, Zhichao

Chen, Zhitong

Cheneler, David

Cheng, Cheng

Cheng, Chin-Chi

Cheng, Fangzhou

Cheng, Fong-Yu

Cheng, Weiying

Cheng, Yang-Tse

Cheng, Yu-Chieh

Cheon, Jason

Cheraghi Bidsorkhi, Hossein

Cheraghian, Goshtasp

Chernyi, Sergei

Chertovskikh, Roman

Chetwynd, Andy

Chew, Nick Guan Pin

Chew, Song Foh

Chiang, Ya-Yu

Chien, Shih-Chang

Chinappi, Mauro

Ching, Congo Tak Shing

Chinnici, Fabio

Chinnici, Marta

Chiriacò, Maria Serena

Chis, Simona

Chiu, Po-Chih

Chiu, Wei-Yu

Chiumenti, Alessandro

Chmielarz, Andrzej

Chmielarz, Lucjan

Cho, Kyungjin

Cho, So-Hye

Cho, Young-Chang

Chobot, Vladimír

Choi, Du-Soon

Choi, Jaeho

Choi, Jane Ru
Choi, Nag Jung

Choi, Pak-Sing

Choi, Sungyong

Choi, Yun Sang

Choiński, Dariusz

Chong, Perk Lin

Chou, Cheng-Tung

Chovan, Tibor

Chowdhury, Dhiman

Chowdhury, Pankaj

Chowdhury, TBM

Christensen, Lars Porskjær

Christina, Spry

Christova, Darinka

Christova-Bagdassarian, Valentina

Chrobok, Anna

Chrysikou, Loukia

$\mathrm{Chu}$, Chun Hung

Chuah, Chong Yang

Chun, Dong Hyuk

Chun, Sejong

Chung, Chan I.

Chung, Haegeun

Chung, Kung-Ming

Chung, Kyeong Woo

Chung, Sheng-Heng

Chwastowski, Jarosław

Chybowski, Leszek

Ciabattoni, Lucio

Ciampi, Simone

Ciceri, Davide

Ciceri, Samuele

Cicero, Sergio

Cicone, Traian

Cid, Clément

Cież, Dariusz

Cipakova, Ingrid

Čiplys, Daumantas

Circiumaru, Adrian

Cirtoaje, Vasile

Čišić, Dragan

Ciski, Mateusz

Cisterna, Pedro

Ciszewski, Mateusz

Claassens, Nico J.

Clematis, Davide

Clément, Nathalie

Climent, Estela

Cloete, Jan Hendrik

Cocconcelli, Marco

Codina, Georgiana 
Coelho, Denis A.

Coelho, Sónia D

Cofelice, Martina

Coffey, William T.

Cohen, Achraf

Cojocaru, Anca

Cojocaru, Corneliu

Colantuono, Antonio

Colavita, Giampaolo

Colella, Carmine

Colla, Valentina

Collivignarelli, Maria Cristina

Colniță, Alia

Colobatiu, Liora

Colomban, Philippe

Colombo, Luigi Pietro Maria

Comuzzi, Clara

Concezzi, Moreno

Conde Cid, Manuel

Conesa, Juan A.

Constantinou, Costas S.

Conterosito, Eleonora

Conti, Fosca

Continillo, Gaetano

Contreras, María Del Mar

Coppo, Massimiliano

Cordioli, Eleonora

Cornejo Garcia, Ivan

Corral Abad, Eduardo

Corrêa, Arlene Gonçalves

Corriou, Jean-Pierre

Corrò, Michela

Corsalini, Massimo

Corsini, Alessandro

Cortaberria, Galder

Cortese, Marta

Cosenza, Maria

Cosgrove, Sebastian

Coskun, Ahmet F.

Cosma, Pinalysa

Cosme, Fernanda

Cossignani, Lina

Costa, Aniko

Costa, Nuno Ricardo

Costa, Rafael S.

Costa, Rui

Costa-Almeida, Raquel

Costantini, Francesca

Costantino, Ferdinando

Costas, Andreea

Costea, Paul Igor
Cote, Danielle

Cotea, Valeriu V.

Couce, María D.

Court, Sébastien

Crauste, Fabien

Cravero, Maria Carla

Cravotto, Giancarlo

Crenganis, Mihai

Cristina, Pardal March

Croizet, Cédric

Crook, Rolf

Cruz, Rebeca

Cruz, Rui M. S.

Cruz, Sandra

Csaki, Stefan

Csordás, Anita

Cucciniello, Raffaele

Cuccurullo, Gennaro

Cuellar, Sara

Cui, Xinjun

Cuibus, Lucian

Cuomo, Francesca

Cupiał, Michał

Curi-Borda, Cecilia K.

Ćurko, Josip

Ćurko, Natka

Ćwieląg-Piasecka, Irmina

Cyplik, Paweł

Cyrus, Václav

Czaja, Paweł

Czarnigowska, Agata

Czerwinski, Robert

Czerwionka, Krzysztof

Czop, Monika

Czosnek, Cezary

D. Patil, Mahesh

D'Adamo, Idiano

D'Ascenzi, Carlo

D'Errico, Gerardino

D'Orazio, Giovanni

Da Silva Dias, Igor Alexandre

Dąbek, Lidia

Dahle, Sebastian

Dai, Jian

Dalaklis, Dimitrios

Dalena, Francesco

Daliakopoulos, Ioannis

Dall'Acqua, Stefano

Dalla Vedova, Matteo D. L.

Dalle Vacche, Sara

Dalmoro, Annalisa 
Damasevicius, Robertas

Dambrauskas, Tadas

Damiano, Fabrizio

Damnjanovic, Ratka

Dańczak, Anna

Daneshgar, Saba

Dani, Samir

Danielak, Dorota

Daniele, Salvatore

Daripa, Prabir

Darmon, Ruxandra

Daroch, Maurycy

Darras, Anastasios

Dascalescu, Lucien

Dashti, Hossein

Dashtian, Hassan

Dátka, Jerzy

Davaritouchaee, Maryam

David, Andrej

David, Laurent

David, Sergio

Davis, Lloyd

Davis, Ryan D.

De Alvarenga, Elson Santiago

De Barros Fernandes, Pedro

De Caroli, Monica

De Carvalho Santos Ebinuma, Valéria

De Corato, Ugo

De Cózar, Abel

De Eugenio, Laura Isabel

De Felice, Fabio

De Gioannis, Giorgia

De Grooth, Joris

De La Hoz, Antonio

De La Piedra, Antonio

De La Rosa, José María

De La Sen, Manuel

De La Vega, José Manuel García

De Lorenzo, Giuseppe

De Marco, Rossella

De Masi, Luigi

De Mingo López, Luis Fernando

De Morais, Sharon D.

De Paepe, Ward

De Rubeis, Tullio

De Sousa Oliveira, Gisela Marta Teixeira

De Sousa, Isabel Maria Nunes

De Spiegelaere, Ward

De Tullio, Mario C.

De Vero, Luciana

De Vita, Daniela
De Wit, Maryna

De, Arijit

Debeleac, Carmen

Dębowski, Marcin

Dębska, Dominika

Dederichs, Anne Simone

Degirmenci, Volkan

Degola, Francesca

Del Giudice, Carmine

Del Hoyo Martinez, Carmen

Delbecq, Frédéric

Delcea, Camelia

Delgado Sobrino, Daynier Rolando

Delgado, Sergio Nogales

Delgado-Bonal, Alfonso

Delichatsios, Michael A.

Delidovich, Irina

Dell' Anna, Maria Michela

Della Torre, Augusto

Dema, Roman

Dembińska, Izabela

Demchuk, Zoriana

Demetrescu, Ioana

Demin, Alexander

Deng, Dongyang

Deng, Tong

Denkova-Kostova, Rositsa Stefanova

Denmark, Daniel J.

Dentcheva, Darinka

Depczynski, Wojciech

Depken, Craig

Deptuła, Milena

Derco, Jan

Deriszadeh, Ali

Deschinkel, Karine

Deshpande, Gauravi

Desplentere, Frederik

Dessi, Claudia

Dewasme, Laurent

Dewberry, Emma

Dewil, Raf

Dhaliwal, Gurjot S.

Dhanasekaran, Saravana M.

Dhar, Bipro R.

Di Blasio, Gabriele

Di Corso, Evelina

Di Felice, Renzo

Di Gravio, Giulio

Di Ilio, Giovanni

Di Lorenzo, Tiziana

Di Martino, Antonio 
Di Masi, Sabrina

Di Mauro, Maria

Di Natale, Concetta

Di Noia, Luigi Pio

Di Nunzio, Mattia

Di Renzo, Alberto

Di Sarli, Valeria

Diaconeasa, Zorita

Diaferia, Carlo

Dias, George J.

Dias, Maria Ines

Díaz De Tuesta, Jose Luis

Diaz Fernandez, Yuri

Diaz, Elena

Diaz, Jose

Díaz-Álvarez, Antonio

Díaz-De Alba, Margarita

Diaz-Perez, Manuel Antonio

Diban, Nazely

Dickert, Franz L.

Dierking, Ingo

Díez, Maria Antonia

Díez-González, Javier

Dikova, Ts. D.

Dimarogona, Maria

Dimitrellou, Dimitra

Dimos, Konstantinos

Dinamarca, Carlos

Dinardo, Francesca

Dindorf, Ryszard

Ding, Wei Dan

Ding, Zhaoyang

Dingley, Andrew J.

Dinica, Rodica-Mihaela

Dinis Gaspar, Pedro

Dinis, Maria Alzira Pimenta

Dinoi, Adelaide

Dionisi, Davide

Dirza, Risvan

Ditta, Allah

Dittmar, Rainer

Divis, Pavel

Dixon, Barry

Djajadi, Demi Tristan

Djeziri, Mohand

Djinović, Petar

Dlauchy, Denes

Dobre, Ciprian

Dobrzanska, Magdalena

Dobrzanski, Pawel

Doctor, Ninad
Doda, Sai Reddy

Dogra, Prashant

Dohi, Toshifumi

Dolfing, Jan

Dołhańczuk-Śródka, Agnieszka

Dolinska, Silvia

Dolocan, Andrei

Domanski, Pawel D.

Domb, Menachem

Domínguez-Amarillo, Samuel

Don, Aakash Welgamage

Donadu, Matthew

Dong, Kejun

Dong, Yiwei

Doni Jayavelu, Naresh

Donovan, John

Doranehgard, Mohammad Hossein

Doretti, Luca

Doria, Enrico

Dorota, Majorkowska-Mech

Dörr, Nicole

Dosta, Joan

Dosta, Maksym

Dostatni, Ewa

Doulgeris, Charalampos

Doustdar, Omid

Dovinova, Ima

Drab, Mitja

Drabińska, Natalia

Dragan, Mirela

Dragomir-Stanciu, Daniel

Drapaca, Corina

Drenkova-Tuhtan, Asya

Drevinskas, Tomas

Drewniak, Józef

Drillet, Jean-François

Drljaca, Miroslav

Drozdov, Andrey

Drożdż, Wioletta

Drużyńska, Beata

Drzazga, Michał

Drzeżdżon, Joanna

Drzymała-Czyż, Sławomira

Duarte, Belmiro

Duarte, Filipe V.

Dubey, Nileshkumar

Dubljevic, Stevan

Dudás, Zoltán

Dudycz, Helena

Duenas, Jose Antonio

Duhamet, Jean 
Dulf, Francisc

Dumele, Oliver

Dumitru, Gabriela

Dummer, Nicholas F.

Dunaj, Paweł

Dundar, Ibrahim

Dung, Jeremiah K. S.

Dunky, Manfred

Durana, Pavol

Durczak, Karol

Durdán, Milan

Đurđević, Boris

Đurin, Bojan

Durmic, Zoey

Dürr, Robert

Dutour Sikirić, Maja

Dutta, Abhishek

Dutta, Sandip

Dwyer, Derek B.

Dwyer, Rocky J.

Dyrba, Martin

Dzida, Marzena

Dzido, Grzegorz

Dziedzic, Krzysztof

Dziki, Dariusz

Dzikuć, Maciej

Dziurkowska, Ewelina

Dzmitry H., Zaitsau

Ebani, Valentina Virginia

Ebrahimiaqda, Elham

Ebrahimzadeh, Amin

Echterhof, Thomas

Eckert, Sven

Eder, Matthias

Edgar, Thomas

Egan, David

Egedy, Attila

Egger, Dominik

Ehrling, Sebastian

Ehsan, Mostafavi

Ein-Mozaffari, Farhad

Eisele, Timothy C.

Eitelberg, Georg

Ekielski, Adam

Elbeaino, Toufic

El-Dars, Farida

Éles, András

El-Gendy, Ahmed A.

EL-Hefny, Mervat

Elias, Jan

Eliseev, Vladimir
Elkasabi, Yaseen

Eller, Zoltán

Ellinas, Kosmas

Ellis, Matthew

Elreedy, Ahmed

Elsaid, Khaled

ElSherbiny, Ibrahim M.A.

Elvas-Leitão, Ruben

EL-Zahar, Essam R.

Elzohairy, Ayman

Emilia, Paone

Endo, Akira

Erdődi, Ferenc

Erenli, Kai

Ermoline, Alexandre

Ernesto-Casanova, Oscar

Eschenbacher, Andreas

Escobedo, Pablo

Escola, José María

Eskin, Michael

Esmaeely Neisiany, Rasoul

Espada-Bellido, Estrella

Espinosa, Eduardo

Estevadeordal, Jordi

Esteves, Ana F.

Esteves, Eduardo

Ettayapuram Ramaprasad, Azhagiya Singam

Etxebeste, Oier

Evans, Patricia

Evon, Philippe

Faccia, Michele

Falsone, Giovanni

Fan, Tai-Hsi

Fandi, Ghaeth

Fandino, Olivia

Fanesi, Marika

Farahani, Behzad V.

Farcas, Anca Corina

Farina, Ilenia

Farinha, José Torres

Farouq Ali, S. M.

Farrokhifar, Meisam

Fatigati, Fabio

Fatouros, Dimitrios G.

Fattah, Islam Md Rizwanul

Fattah, Kazi Parvez

Fauzi, Rizal Taufiq

Favaloro, Anthony

Favre-Réguillon, Alain

Fawell, Phillip

Fazal, Zeeshan 
Fedeli, Paolo

Fediuk, Roman

Feduraev, Pavel

Felde, Imre

Feng, Yin

Feng, Yinnian

Ferdes, Mariana

Ferdinandy, Bence

Ferella, Francesco

Ferenc, Kristály

Ferlazzo, Nadia

Fermeiro, J. B. L.

Fernandes, Maria C.

Fernández Ochoa, Álvaro

Fernandez Oro, Jesus Manuel

Fernández, Antonio

Fernández, Carlos Vilas

Fernández, José A.

Fernández, María García

Fernandez-Gamiz, Unai

Fernandez-Lafuente, Roberto

Fernández-Lucas, Jesús

Ferone, Mariateresa

Ferrández, Daniel

Ferrari, Michele

Ferraro, Vincenza

Ferreira Barroso, Ana Paula

Ferreira, Bárbara

Ferreira, Carlos Silva

Ferreira, Joao C.

Ferreiro-González, Marta

Ferrero Aguar, Pablo

Ferretti, Ivan

Ferri, Nicola

Ferry, Laurent

Fesl, Jan

Feucherolles, Maureen

Fidelibus, Corrado

Fiebig, Wiesław

Fievet, Patrick

Figueira, Ana Cristina

Fike, Matej

Filip, Bogdan Florin

Filip, Petr

Filipeanu, Catalin

Findorák, Róbert

Findura, Pavol

Finimundy, Tiane C.

Fiorineschi, Lorenzo

Fister, Dušan

Fitzpatrick, John
Flamm, Christoph

Fletcher, Ashleigh

Fletcher, Jane

Flieger, Jolanta

Flizikowski, Jozef

Flores Johnson, Emmanuel Alejandro

Florescu, Adriana

Floresta, Giuseppe

Florimond, Guéniat

Floris, Barbara

Flotats, Xavier

Fodor, Marietta

Fodor, Petru S.

Fogarasi, Szabolcs

Fomenko, Anton

Fomin, Alexey

Fonlupt, Cyril

Foresi, Gabriele

Formela, Krzysztof

Foscolo, Pier Ugo

Fouatih, Omar Madani

Fourlas, George K.

Frąckiewicz, Mariusz

Fragomeni, Gionata

Fragos, Vassilios

Framinan, Jose M.

Franceschini, Giordano

Franch, Gabriele

Franco Martínez, Juan Agustín

Franco, Alessandro

Franco-Duarte, Ricardo

François Fréty, Roger Thomas

François, Gregory

Franczyk, Adrian

Frankcombe, Terry J.

Franke, Knut

Franken, Tanja

Frankowski, Jakub

Frass, Michael

Freddi, Luca

Freimoser, Florian

Freire, Ana Cristina

Freire, M. Sonia

Frerich, Sulamith C.

Friedrich, Jan

Frolov, Andrej

Frontera, Patrizia

Frontistis, Zacharias

$\mathrm{Fu}$, Guopeng

$\mathrm{Fu}, \mathrm{Li}$

$\mathrm{Fu}$, Xinyu 
Fuertes-Miquel, Vicente S.

Fügenschuh, Armin

Fugol, Małgorzata

Fukuda, Mitsuru

Fukushima, Atsushi

Fulignati, Sara

Fumagalli, Laura

Funazukuri, Toshitaka

Fung, Rong-Fong

Funk, Richard

Funke, Axel

Fuoco, Tiziana

Furgal, Joseph

Furlani Bastos, Alvaro

Furmańczyk, Konrad

Furukawa, Kenji

Furuta, Hiroshi

Fusco, Giulio

G. Kokotou, Maroula

Gaballo, Antonio

Gaber, Timo

Gabl, Roman

Gábor, Szepesi L.

Gabriela, Plaiasu

Gabrielli, Fabrizio

Gac, Wojciech

Gaglione, Rosa

Gago, Sandra

Gaj, Kazimierz

Gajdosik, Martina Srajer

Gajewska, Magdalena

Gala, Rikhav P.

Galamboš, Michal

Galan, Ana-Maria

Galán, Miguel Ladero

Galanakis, Charis

Galán-Casado, Santos

Galli, Federico

Galli, Viola

Gallo, Monica

Gallucci, Francesco

Galushkin, Alexander A.

Gałuszka, Adam

Galvanin, Federico

Gambuti, Angelita

Gamez Montero, Pedro Javier

Gan, Samuel

Ganapathi Shanmugam, Shankar

Gandolfi, Marco

Gani, Rafiqul

Gantner, Magdalena
Gao, Jianbing

Gao, Jun

$\mathrm{Gao}, \mathrm{Xu}$

Gao, Yuesheng

Gao, Zhiye

Garbayo, Inés

Garbowska, Monika

Garcia Cascales, Maria Socorro

García Costa, Alicia Loreto

García, David Marín

García, Juan T.

García, Lucía

García, Marcelo

García, Miguel Torres

Garcia, Rosario

Garcia-Baños, Beatriz

García-Delgado, Carlos

Garcia-Llatas, Guadalupe

García-Ordás, María Teresa

García-Ruiz, Almudena

Gardeli, Chrysavgi

Gardiner, Philip

Gardini, Davide

Gargiulo, Massimiliano

Gargiulo, Valentina

Garmendia, Iñaki

Garncarek, Zbigniew

Garofalo, Emilia

Gaska, Krzysztof

Gatchell, Michael

Gautam, Siddharth S.

Gavalas, Evangelos

Gavan, Alexandru

Gaweł-Bęben, Katarzyna

Gebremariam, Shemelis Nigatu

Géczy, Attila

Geem, Zong Woo

Geletii, Yurii

Genova, Chiara

Genovese, Giuseppa

Genovese, Maria Erminia

Gentile, Gennaro

Gentili, Denis

George, Thomas F.

Georgescu, Andrei-Mugur

Geraldes, Vitor

Gerasimov, Evgeniy Yu.

Gerislioglu, Burak

Gerke, Jörg

Gesell, Andreas

Geszke-Moritz, Małgorzata 
Gevari, Moein Talebian

Ghandehari, Mehran

Ghazi-Nezami, Farnaz

Ghedira, Sami

Gheju, Marius

Gheorghe, Dumitrascu

Ghidelli, Matteo

Ghorbani, Morteza

Ghosh, Abhishek

Ghosh, Kanka

Ghosh, Raja

Ghosh, Santosh

Ghosh, Soumita

Giacometti, Andrea

Giacosa, Simone

Giammanco, Anna

Giannakakis, Giorgos

Giannakeas, Nikolaos

Gianni, Areti

Gibson, Christopher

Gidon, Dogan

Gierszewski, Mateusz

GIl, Bartosz

Gil, Nuno

Gil, Stanisław

Gilassi, Sina

Gill, Rajan

Gille, Andrea

Giménez-Gómez, Pablo

Giorcelli, Mauro

Giorgio, Guariso

Giorno, Lidietta

Girard, Etienne

Gîrțu, Mihai A.

Gisbert-Garzarán, Miguel

Gitlin-Domagalska, Agata

Giuffrè, Angelo Maria

Giuffrida, Paolo

Giuggioli, Nicole Roberta

Giuliani, Chiara

Giuliano, Aristide

Giunta, Marinella

Giuranno, Donatella

Gjelseth Antonsen, Simen

Gkillas, Konstantinos

Glasmacher, Birgit

Glasser, Leslie

Gligor, Adrian

Gligor, Delia Maria

Gliniak, Maciej

Gloess, Alexia N.
Głowacki, Tadeusz

Gluck, Jessica

Glynn, Peter

GNATOWSKA, Renata

Gnatowski, Andrzej

Göcsei, Gábor

Godeau, Guilhem

Godina, Radu

Godyń, Katarzyna

Goel, Himanshu

Gogolewski, Damian

Gohari, Soheil

Gołaszewski, Janusz

Goldrick, Stephen

Golmohamadi, Hessam

Golubnitschaja, Olga

Gombár, Miroslav

Gomes, Ana P.

Gomes, Ana T. P. C.

Gomes, Carlos Leal

Gomes, Joao

Gomes, Joao F

Gómez Barrios, Xiomar

Gómez, Manuel Checa

Gómez-Hernández, Jesús

Gomolka, Zbigniew

Goncalves, Olivier

González Briones, Alfonso

GOnzalez Castano, Miriam

González García, Mariano

Gonzalez Viejo, Claudia

González, Enrique

González, Javier Serrano

González, Jose

Gonzalez-Alvarez, Isabel

González-De-Peredo, Ana V.

González-Portillo, Luis F.

González-Sánchez, Rocío

Gorbanev, Yury

Gore, Ross

Górniak, Aleksander

Górnicki, Krzysztof

Gorodkiewicz, Ewa

Goroncy, Alexander

Gorri, Daniel

Górska, Agata

Gościańska, Joanna

Gospodarek, Janina

Gostomski, Peter

Goti, Aitor

Goto, Kazuya 
Goto, Satoru

Gotoh, Hiroaki

Gotor-Fernández, Vicente

Gottschalk, Axel

Gotzias, Anastasios

Goudoulas, Thomas

Goula, Maria A.

Gourinat, Yves

Govan, Joseph

Grabarczyk, Robert

Grabowska, Sandra

Grab-Rogaliński, Karol

Graczová, Elena

Gramza-Michałowska, Anna

Grandhi, Srimannarayana

Granjo, Jose

Grassi, Alfonso

Grasso, Marzio

Grau, Etienne

Gray, Murray

Graziani, Gabriela

Grbes, Anamarija

Grbić, Ratko

Grbovic, Dragoslav

Greco, Adriana

Grecov, Dana

Greetham, Darren

Gregori, Alberto

Greiner, David

Grgas, Dijana

Grizzuti, Nino

Groch, Paweł

Grosser, Anna

Grossi Bovi Karatay, Graziele

Groves, Matthew

Grubecki, Ireneusz

Gruszka, Konrad

Grzegorzek, Martyna

Grznár, Patrik

Gu, Fengshou

Gualtieri, Carlo

Guan, Zeyi

Guarino, Stefano

Gubiani, Rino

Gubitosa, Jennifer

Guedens, Wanda

Guedes, Rita C.

Guedes-Alonso, Rayco

Guérardel, Yann

Guerrieri, Nicoletta

Guida, Giulia
Guida, Marco

Guijt, Rosanne M.

Guizzi, Guido

Gullo, Paride

Gulson, Brian

Gumbytè, Milda

Guminska, Jolanta

Gun'ko, Vladimir M.

Gunduz, Seval

Gunkelmann, Nina

Gunputh, Urvashi

Guo, Dandan

Gupta, Charu

Gupta, Murlidhar

Gutierrez, Junkal

Gutiérrez-Arzaluz, Mirella

Guyoneaud, Rémy

Guziałowska-Tic, Joanna

Guzmán, Eduardo

Guzzon, Antonella

Gyurkó, Zoltán

Ha, Don-Hyung

Ha, Hoehun

Habashi, Fathi

Haber, Rodolfo E.

Habuda-Stanic, Mirna

Habuda-Stanić, Mirna

Hadjikakou, Sotiris

Haguet, Vincent

Hahn, Jae Ryang

Hailu, Getu

Hajduga, Arkadiusz

Hajilou, Mohammadhadi

Hajiyev, Natig Qadim-Oglu

Hájková, Lenka

Halabalaki, Maria

Halilovič, Miroslav

Hamdani, Ari

Hamdy, Louise

Hamedi, Hamid Reza

Hamon, Morgan

Han, Seong-Ik

Hanamoto, Seiya

Hanania, Michel

Hanaoka, Toshiaki

Handa, Taro

Haneklaus, Nils

Hangan, Anca

Hanik, Nils

Hanke, Tomas

Hanks, Timothy 
Hannides, Angelos

Hansen, Anders Hedegaard

Haque, Md Emdadul

Harabaglia, Paolo

Harcum, Sarah

Harhar, Hicham

Harker, Anthony

Harkess, Richard

Harper, Jason B.

Hart, Abarasi

Harvel, Glenn

Harvianto, Gregorius

Hasan, Mohammad H.

Hashem, Tawheed

Hashim, Khalid

Hassan, Amro B.

Hassan, Md. Kamrul

Hassan, Sammer-ul

Hassanzadeh-Barforoushi, Amin

Hastings, Astley

Hatami, Mohsen

Hategan, Camelia Daniela

Hatzilyberis, Kostas S.

Haupt, Michael

Hausmann, Joachim

Havlík, Jan

Hay, Thomas

Hayashi, Yohei

Haybaeck, Johannes

Haydary, Juma

Hazai, László

He, Guanjie

He, Jiarui

He, Wei

He, Wenhan

He, Yuxin

Hecel-Czaplicka, Aleksandra

Heger, Dominik

Heidarinejad, Mohammad

Heierli, Joachim

Hein, Kyaw

Heinlein, Alexander

Helle, Mikko

Heltzel, Jacob

Hemmerich, Johannes

Hendrick, Patrick

Henen, Morkos A.

Hennig, Sven

Henriques, João

Hensley, Alyssa

Heo, Man-Woong
Herak, David

Heras, Francisco

Herbert, John

Hernández Del Olmo, Félix

Hernández, Luis $M$.

Hernandez-Aldave, Sandra

Hernandez-Bautista, Rene

Hernandez-Vazquez, Jesus-Maria

Herrera, Manuel

Herrera-Melián, José

Herreros López, Alberto

Herrmann-Geppert, Iris

Herterich, James

Herth, Etienne

Hettiarachchi, Hiroshan

Heydari, Azim

Heyder, Rodrigo

Hidalgo Herrador, José Miguel

Hideki, Kurokawa

Hildner, Richard

Hill, Janet

Hiller, Sven-Jürgen

Hinestroza, Juan

Hinojosa, Victor $\mathrm{H}$.

Hinrichs, Wouter L. J.

HIROKI, UCHIYAMA

Hirose, Sohichi

Hirschnitz-Garbers, Martin

Hlava, Jaroslav

Hlaváč, Libor M.

Hnatiuc, Mihaela

Ho, Chia-Che

Ho, Danny C. K.

Ho, Jia Shin

Ho, Jörg

Ho, Long

Hoadley, Andrew

Hoaghia, Alexandra

Hoff, Craig J.

Hofman, Tadeusz

Hohenegger, Martin

Holappa, Lauri

Holmberg, Henrik

Holst, Anders

Holuszko, Maria E.

Hołyńska-Iwan, Iga

Honda, Masaki

Honda, Shunichi

Hong, Cheng-Yih

Hong, Seock-Jin

Hong, Seok Hoon 
Hong, Seungbum

Hong, Soon-Jik

Hönig, Vladimír

Hontoria, Leocadio

Horabik, Józef

Horai, Yoshiro

Horchani, Habib

Horník, Miroslav

Horovistiz, Ana

Hortigón-Vinagre, María

Horvat, Barbara

Hős, Csaba

Hosomi, Ryota

Hossain, A. S. M. Monjur Al

Hossain, Abul

Hossain, Md Shakhawath

Hossain, Md. Alamgir

Hosseini Boosari, Seyed Sina

Hosseinian-Far, Amin

Hou, Jingwei

Hovde, Blake T.

Howsmon, Daniel

Hrobárik, Peter

Hroboňová, Katarína

Hrustić, Enis

Hryniewicz, Olgierd

Hsieh, Lu-Sheng

Hsu, Hsien-Pin

Hsueh, Hsin-Ta

Hsueh, Yi-Huang

$\mathrm{Hu}$, Anming

$\mathrm{Hu}$, Chenlin

$\mathrm{Hu}$, Guoli

$\mathrm{Hu}$, Guoping

$\mathrm{Hu}$, Maocong

$\mathrm{Hu}$, Wanhe

$\mathrm{Hu}$, Weichih

$\mathrm{Hu}$, Xiaozhi

$\mathrm{Hu}$, Zhigang

Hua, Jie

Huang, Chih-Feng

Huang, Chung-Jen

Huang, Genin Gary

Huang, Guosheng

Huang, Hsiang-Hsi

Huang, Huachao

Huang, Hung Ji

Huang, Ko-Wei

Huang, Pei-Chi

Huang, Sheng-Hsiu

Huang, Wu-Jang
Huang, Xiaowu

Huang, Yifeng

Huang, Yu-Chuen

Huang, Yuhan

Huang, Zhuangrong

Hubenova, Yolina

Huber, Robert

Huber, Roland G.

Hubicka, Urszula

Hübner, Florian

Hudak, Paul

Hudon, Nicolas

Hughes, Jeff

Huh, Taewook

Humelnicu, Doina

Hung, Weihsi

Hung, Yung-Tse

Huo, Jiajie

Hussain, Suhail S. M.

Hwang, Jiun-Ren

Hwang, Yuhoon

Hwangbo, Hyunwoo

Hýsek, Štěpán

Hyunik, Yang

Ibl, Martin

Ibrahim, Salam

Ibrar, Ibrar

Idda, Maria Laura

Ieracitano, Cosimo

Ifelebuegu, Augustine

Iglesias, Raquel

Igliński, Bartłomiej

Igreja, José

Igual, Jorge

Iijima, Kazutoshi

Ikeda, Hiroshi

Ikram, Awais

Ileana Corcau, Jenica

Iliescu, Mariana

Iliev, Boyan

Ilinčić, Petar

Iluk, Artur

Im, Hyungsoon

Imai, Jun

Imaz, Inhar

Imperatore, Concetta

Imran, Muhammad

Ingaldi, Manuela

Ingram, Andy

Ingram, Conrad

Innocenzi, Valentina 


\author{
Inoue, Seiji \\ Ioan-Lucian, Popa \\ Ioannou, Stelios \\ Ion, Rodica-Mariana \\ Ion-Ebrasu, Daniela \\ Ionescu, Clara \\ Ionescu, Mihaela Ileana \\ Ionescu, Mihail \\ Ionita, Petre \\ Iovieno, Michele \\ Ipsakis, Dimitris \\ Iqbal, Hafiz M. N. \\ Iqbal, Kamran \\ Iqdiam, Basheer \\ Irannejad, Abolfazl Mani \\ Iranzo, Alfredo \\ Iris, Cagatay \\ Irshad, Ahamed \\ Iseppi, Ramona \\ Ishigaki, Masahiro \\ Iskander, Syeed Md \\ Islam, Md. Rashedul \\ Islam, Sheikh \\ Isola, Gaetano \\ Isvoranu, Dragos \\ Ito, Kazuhide \\ Ivanov, Vladimir K. \\ Ivanowa, Tatiana \\ Ivascu, Larisa \\ Ivchenko, Pavel \\ Iwaniszyn, Marzena \\ Izawa, Hironori \\ Izumi, Atsushi \\ J. Jankiewicz, Bartłomiej \\ J. Jewiarz, Marcin \\ Jabbari, Faryar \\ Jablan, Jasna \\ Jadidi, Mehdi \\ Jadlovska, Slavka \\ Jafarinejad, Shahryar \\ Jagatić Korenika, Ana Marija \\ Jagtap, Sujit \\ Jahandar Lashaki, Masoud \\ Jahangiri, Leila \\ Jahromi, Hossein \\ Jakobsen, Hugo \\ Jakobusic Brala, Cvijeta \\ Jakubik, Wiesław P. \\ Jakubowski, Marek \\ Jalowiecki-Duhamel, Louise
}

Jamalabadi, Mohammad Yaghoub

Abdollahzadeh

Jamil, Mohsin

Jammalamadaka, Udayabhanu

Jamróz, Dariusz

Jamróz, Ewelina

Jan, Heiland

Janagam, Dileep Reddy

Janas, Dawid

Janecek, Stefan

Janiszewska-Turak, Emilia

Jankovic, Brankica

Jankowiak, Corentin

Jankowski, Łukasz

Janošovský, Ján

Janus, Rafał

Januševičius, Karolis

Januszewicz, Katarzyna

Jaoul, Cédric

Jaroszewska, Karolina

Järvinen, Mika

Jasinski, Jaroslaw Jan

Jasiński, Marek

Jasinski, Michal

Jasiulewicz-Kaczmarek, Malgorzata

Jasiunas, Lukas

Jaśkowska, Jolanta

Jaskulski, Roman

Jasutiene, Ina

Jayant, Rahul

Jedynak, Katarzyna

Jeffryes, Clayton

Jeffs, James

Jemrić, Tomislav

Jeng, Dong-Sheng

Jensen, Svend Borup

Jeon, Heung Seok

Jeon, Seog-Jin

Jeong, Jaewook

Jeong, Minjoong

Jeong, Yoonah

Jerman, Andrej

Jermy, Mark

Jerzak, Wojciech

Jeux, Victorien

Jezierski, Jan

Jheng, Yu-Ming

Ji, Sungchul

Jia, Bao

Jia, Xiaodong

Jiang, Liangliang 
Jiang, Wei

Jibrin, Abubakar Abbas

Jiménez, Jorge Cara

Jimenez-Gomez, Alejandro

Jimenez-Hornero, Jorge E.

Jin, Shengli

Jirout, Tomas

Jiroutová, Dita

Jishkariani, Davit

Jo, Byung Hoon

Jo, Young Min

Johnson, David

Johnston, Nigel

Jonsson, Sofi

Jorat, Ehsan

Jordan, Christian

Jørgensen, Sten Bay

Jornaz, Abdelmonaem

Jose Francisco, Jose Francisco

Joshaghani, Alireza

Joshi, Sameer

Joshi-Navare, Kasturi

Jothi, Palani Raja

Józsa, Viktor

Jradi, Khalil

Juaidi, Adel

Juchelkova, Dagmar

Jugessur, Aju

Juhász, Réka

Juhna, Talis

Jukić Špika, Maja

Juknelevičius, Romualdas

Julio, Bastos Arrieta

Juneja, Ankita

Jung, Hyun Wook

Jung, Seunho

Juniper, Matthew

Junne, Stefan

Jurasekova, Zuzana

Jůza, Josef

K. Hassan, Mohammad

Kaario, Ossi

Kabala, Zbigniew

Kabir, Humayun

Kačániová, Miroslava

Kacem, Mariem

Kačur, Ján

Kaczmarczyk, Jarosław

Kaczmarek, Beata

Kaczmarek, Michal

Kaczmarek, Slawomir M.
Kádár, Csilla

Kadnár, Milan

Kaerger, Joerg

Kager, Julian

Kahlert, Heike

Kaján, Győző L.

Kakar, Farokh Laqa

Kako, Tetsuya

Kalathiya, Umesh

Kale, Manoj

Kalenik, Marek

Kaliniewicz, Zdzisław

Kalinowska Lis, Urszula

Kalinowski, Krzysztof

Kalla, Smail

Kalligeros, Stamatis Spyridon

Kalogiouri, Natasa

Kam, Antony

Kam, Rothman

Kamaltdinov, Vyacheslav

Kamanina, Natalia

Kaminski, Daniel M.

Kaminsky, Heather

Kamp, Christel

Kamperidou, Vasiliki

Kan, Chi-wai

Kanaujiya, Jitendra

Kanbur, Barış Burak

Kancharla, Abhilash

Kancherla, Raghu

Kandylis, Panagiotis

Kaneda, Masayuki

Kang, CongBao

Kang, Hokeun

Kang, Jonghoon

Kang, Ki Hyuk

Kang, Moon-sung

Kantak, Chaitanya

Kantor, Ivan Daniel

Kantzas, Apostolos

Kanzaki, Yoshiki

Kapranov, Sergey

Kapuśniak, Janusz

Kapusta, Ireneusz

Kapusta, Łukasz Jan

Karakalas, Anargyros

Karakashev, Stoyan

Karakasidis, Theodoros

Karakatič, Sašo

Karakoç, Alp

Karampour, Hassan 
Karasali, Helen

Karaśkiewicz, Krzysztof

Karayiannis, Dimitrios

Karbwniczek, Miroslaw

Karcz, Dariusz

Kardas, Dariusz

Kardas, Edyta

Karimi, Sajjad

Karkalos, Nikolaos E.

Karlis, Athanasios

Karlsson, Stefan

Karolewicz, Bożena

Karras, Panagiotis

Karvelas, Evangelos

Kasai, Fumio

Kasimanickam, Vanmathy R.

Kaškonienė, Vilma

Kasperski, Andrzej

Kassai, Miklós

Kästner, Christian

Kathe, Mandar

Kathuria, Himanshu

Katinas, Egidijus

Kato, Hironori

Katrib, Juliano

Katryniok, Benjamin

Katsimpouras, Constantinos

Katz, Jonathan I.

Kaup, Magdalena

Kavousanakis, Mihalis

Kawaguchi, Misa

Kawahara, Jun

Kawakita, Hidetaka

Kayaki, Yoshihito

Kazakova, Oxana B.

Kazamer, Norbert

Kazarin, Petr

Kaziev, Andrey V.

Kazimierowicz, Joanna

Каледина, Нина

$\mathrm{Ke}$, Xie

Kechagias-Stamatis, Odysseas

Kee Huong, Lai

Keerio, Hareef Ahmed

Keipert, Christine

Kelemen, Miroslav

Kelley, Steven

Kepinski, Leszek

Kerdouss, Fouzi

Kerkhoven, Eduard

Keshavarzian, Sajjad
Keshwani, Deepak

Khakalo, Sergei

Khalighi, Sanaz

Khan, Inayat Ali

Khan, Irfan Ahmad

Khan, Muhammad

Khan, Omar F.

Khan, Samir

Khandaker, Morshed

Khang, Gilson

Khanongnuch, Ramita

Kharrat, Riyaz

Khattab, Muhammad

Khattab, Tawfik

Khodadadi, Jay M.

Khodadadi, Mohammad

Khojasteh Salkuyeh, Yaser

Khond, Vivek W.

Khovanov, Igor

Khura, Maninder

Kida, Małgorzata

Kieliszek, Marek

Kikuchi, Ryuji

Kikuchi, Takashi

Kim, Daehwan

Kim, Eun-Seok

Kim, Hanki

Kim, Han-Shin

Kim, Hoe Joon

Kim, Hyun Goo

Kim, Hyungmin

Kim, Hyunju

Kim, Jae Chang

KIM, Jeong Soo

Kim, Jin Sik

Kim, Jong-Chan

Kim, Junbom

Kim, Junil

Kim, Keun-Sik

Kim, Ki Su

Kim, Kibum

Kim, Kiyoung

Kim, Min-Cheol

Kim, Min-Suk

Kim, Seung Wook

Kim, Sok

Kim, Su-Hyeon

Kim, Sung Kyeom

Kim, Sung Won

Kim, Yang

Kim, Young-Kee 
Kim, Youngmee

King, Maria

Kiokias, Sotirios N.

Kioumarsi, Mahdi

Kiparissides, Costas

Kirilov, Plamen

Kirner, Raimund

Kisiel, Tomasz

Kiss, Imre

Kiss, János

Kita, Agnieszka

Kitagawa, Toshiaki

Kitrytè, Vaida

Kitukutha, Nicodemus

Klanšek, Uroš

Klaučo, Martin

Kleimt, Bernd

Klemes, Jiri

Klewicka, Elzbieta

Klimczak, Marek

Klimecka-Tatar, Dorota

Klimek, Anna

Kłos, Marcin

Kłosowski, Grzegorz

Kmetič, Ivana

Kminiak, Richard

Knap, Matjaž

Knupp, Gerd

Knupp, Kevin R.

Ko, Chun-Han

Kobayashi, Motoyoshi

Kobeissy, Firas

Kobrak, Mark N.

Kobus, Zbigniew

Koch, Wojciech

Koda, Eugeniusz

Koga, Shiori

Koilakos, Dimitrios I.

Kojima, Chojiro

Kokkinos, Nikolaos C.

Kollmann, Wolfgang

Kolniak-Ostek, Joanna

Kołodyńska, Dorota

Koltsaklis, Nikolaos

Komba, Shiro

Komorek, Andrzej

Komorska, Iwona

Koncsag, Claudia Irina

Kondaveeti, Sanath

Kondinski, Aleksandar

Kong, Xiangrui
Kongar, Elif

Konopacka-Łyskawa, Donata

Konstanciak, Anna

Konstandinidou, Myrto

Konstantynowicz-Nowicka, Karolina

Konstas, Kristina

Konsulova-Bakalova, Mariya

Kontoravdi, Cleo

Koolivand, Abdollah

Koomullil, Roy P.

Koopmans, Lucien

Kopecký, Dušan

Kopel, Pavel

Koppen, Mario

Kopsahelis, Nikolaos

Kore, Rajkumar

Korlos, Apostolos

Korobiichuk, Igor

Korzeniowska, Malgorzata

Kosel, Janez

Kosieradzka, Anna

Kost, Bartłomiej

Köstler, Stefan

Kostoglou, Margaritis

Kostrzewa, Daniel

Koswara, Andy

Koszela, Krzysztof

Kot, Anna

Kotcon, James B.

Kotteda, V M Krushmarao

Kotula-Balak, Małgorzata

Kotwica, Krzysztof

Kotwicki, Lech

Kotzamanidis, Charalambos

Kourmatzis, Agisilaos

Kouziokas, Georgios N.

Kouzu, Masato

Kovačič, Miha

Kovářr, Jiř́i

Kovari, Attila

Kowalczuk, Dorota

Kowalczuk, Przemyslaw

Kowalczyk-Juśko, Alina

Kowalska, Ewa

Kowalska, Hanna

Kowalska, Jolanta

Kowalski, Gregory

Kozai, Toyoki

Kozieł, Edmund

Kozielski, Lucjan

Kozioł, Joachim 
Kozlov, Alexander

Kozlova, Ekaterina

Kozma, Gábor

Kozub, Barbara

Kracík, Petr

Krajcovic, Martin

Krajka-Kuźniak, Violetta

Král, Petr

Kralj, Samo

Kramlich, John C

Kratochvilova, Irena

Kraut, Manfred

Krawczyk, Łukasz

Krawczynski, Kamil

Krebsz, Melinda

Křen, Vladimir

Krimi, Abdelkader

Krischek, Carsten

Krishnan, Vaishakh

Krishnasamy, Gopinath

Kristensen, Erik Fløjgaard

Kritis, Aristeidis

Krivoruchko, Anastasiia

Krokidis, Marios

Król, Danuta

Król, Ewelina

Król-Kilińska, Żaneta

Kroner, Anna B.

Krupińska, Izabela

Krzanowski, James

Krzebietke, Sławomir Józef

Krzemianowski, Zbigniew

Krzywda, Roman

Krzywonos, Małgorzata

Krzyzynska, Renata

Kubiak, Katarzyna

Kubiak, Piotr

Kubica, Paweł

Kubík, Lubomír

Kučera, Lukáš

Kučera, Marián

Kucerova, Marta

Kucharska, Barbara

Kucharska, Karolina

Kuczyńska, Beata

Kudasik, Mateusz

Kudelas, Dušan

Kudlek, Edyta

Kudo, Shinji

Kudr, Jiri

Kuenz, Anja
Kuever, Jan

Kühl, Hans-Detlev

Kujawa, Joanna

Kujawska, Justyna

Kukula-Koch, Wirginia

Kukulka, David J.

Kulak, Michał

Kułażyński, Marek

Kulczycki, Andrzej

Kulesha, Olga

Kulesza, Zbigniew

Kulišić, Biljana

Kulkarni, Shashank

Kumano, Teruhisa

Kumar, Rajeev

Kumar, Vikas

Kumar, Vineet

Kumhála, František

Kun, Ádám

Kung, Yu-Chun

Kungolos, Athanasios

Kunli, Goh

Kunugi, Tomoaki

Kuo, Chia-Hung

Kuo, Meng-I (Marie)

Kuo, Tsung-Rong

Kurasiak-Popowska, Danuta

Kurihara, Satoshi

Kurkus-Gruszecka, Michalina

Kursun, Celal

Kurt, Mehmet

Kus, Piotr

Kusano, Kanichi

Kusch-Brandt, Sigrid

Kushnir, Sergey E.

Kušnerová, Milena

Kustov, Sergey

Kusyumov, Alexander

Kuszewski, Hubert

Kuti, Olawole

Kuts, Vladimir

Kuttner, Christian

Kuzhiumparambil, Unnikrishnan

Kuzmina, Ksenija

Kuźniar, Agnieszka

Kuźnik, Nikodem

Kwade, Arno

Kwak, Jin Kyung

Kwapinska, Marzena

Kwapiszewska, Karina

Kwiatkowski, Mirosław 
Kwiatoń, Paweł

Kwon, Beomjin

Kwon, Gibum

Kwon, Joseph Sang-Il

Kwon, Yongchan

Kwon, Young M.

Kyprianidis, Konstantinos

Kyriakopoulou, Konstantina

Kyzas, George Z.

La Rocca, Michele

Lach, Ralf

Lachowicz, Sabina

Laciak, Marek

Lackner, Maximilian

Lacrampe, Marie-France

Ladaci, Samir

Lado, Rita

Laganà, Antonio Simone

Laham-Karam, Nihay

Lai, Stefano

Lai, Wing-Fu

Lamas, María Isabel

Lamb, Jacob J.

Lamponi, Stefania

Lang, Peter

Langer, Jerzy J.

Lankester, Felix

Lanotte, Ruggero

Łapczyńska-Kordon, Bogusława

Lapenna, Pasquale Eduardo

Łapiński, Marcin

Łapka, Piotr

Lapkovskis, Vjaceslavs

Lapointe, Mathieu

Lappalainen, Katja

Lapushkina, Tatiana

Laronze-Cochard, Marie

Larson, Nicholas B.

Larsson, Simon

Larumbe, Silviax

Larwa, Barbara

Lasik-Kurdyś, Małgorzata

Łaskawiec, Edyta

Laskowska, Magdalena

Latosińska, Jolanta

Lau, Tiffany

Lauberts, Maris

Laura, Romero-Zeron

Laurent-Applegate, Lee Ann

Lauret, Pierre

Laurila, Hannu
Lavano, Angelo

Lawn, Chris

Lay, Chyi-How

Lazzara, Giuseppe

Lazzari, Massimo

Le Normand, Francois

Le Thanh-Blicharz, Joanna

Le, Hoang-Thanh

Le, Tien Loc

Leão, Celina

Lebaz, Noureddine

Lebihan, Yann

Leckner, Bo

Lederer, Franziska L.

Ledgerwood, Elizabeth C.

Lee, Byung Joon

Lee, Changgu

Lee, Changhee

Lee, Chi-Guhn

Lee, Dongheon

Lee, Gil-Yong

Lee, Harry F.

Lee, Hunjoo P.

Lee, Hyoung Jin

Lee, Jaesung

Lee, Jechan

Lee, Jen-yao

Lee, Jeongmi

Lee, Jihyun

Lee, Jin Hyung

Lee, Joon Kyu

Lee, Kihyung

Lee, Kiwon

Lee, Moonyong

Lee, Myung-Chul

Lee, Ping-Hui

LEE, Sang Wook

Lee, Sanghoon

Lee, Seung

Lee, Seung-Min

Lee, Shie-Jue

Lee, Sihyun

Lee, Sunjae

Lee, Tae-Kwon

Lee, Won-Ju

Lee, Ye-Eun

Lehocky, Marian

Lei, Fan

Leitão, Jorge H.

Leitgeb, Maja

Lejman, Agnieszka 
Lekunberri, Edorta Carrascal

Lemanowicz, Marcin

Lemli, Beata

Lemos, Maria Amélia N. D. A.

Lenci, Alessandro

Lenci, Elena

Lengani, Davide

Lenhart, Suzanne

Lennartsson, Andreas

Lenzo, Basilio

Leon, Francisco

León, Gerardo A.

Léonard, Grégoire

Leonov, Sergey B.

Leonow, Sebastian

Leontopoulos, Stefanos

Leonzio, Grazia

Lerch, Andre

Leskarac, Domagoj

Lesnik, Luka

Lesyk, Roman B.

Levesque, Dominique

Lewandowicz, Jacek

Lewinska, Agnieszka

Lewinska, Anna

Lexa, Matej

Leybo, Denis

Li, Chengxi

Li, Chuan

Li, Guohui

Li, Hao

Li, Hongyang

Li, Jie

Li, Ji-Qin

$\mathrm{Li}$, Lin

Li, Mingheng

Li, Sipei

Li, Siyi

Li, Tomi T.

Li, Xiang

Li, Xiang-Guo

Li, Xue

Li, Ying

Li, Zhongliang

Li, Zidong

Liang, Chao

Liang, Dawei

Liang, Wenkai

Liao, Chien-Sen

Liao, Chun-Chung

Liao, Hung-Ju
Liauzun, Cedric

Licciardello, Feliciana

Licciardi, Silvia

Liechti, George W.

Liesiene, Jolanta

Lignos, Ioannis

Lila, Mary Ann

Lim, Chun Shen

Lim, Dae-Eun

LIM, Gilbert

Lim, Ocktaeck

Lima, Dênis

Lin, Cherng-Yuan

Lin, Jingyu

Lin, Mingzhe

Lin, Po-Heng

Lin, Sidney

Lin, Wei-Chao

Lin, Weifeng

Lin, Yangju

Linares, María

Lindemann, Peter

Lindholm-Lehto, Petra

Ling, Shengjie

Lintner, Karl

Lio, Wai Hou

Liobikas, Julius

Lionetti, Vincenzo

Liou, Bo-Kang

Lipiński, Adam

Lipscomb, Glenn

Lis Arias, Manuel J.

Lisowski, Aleksander

Lisowski, Edward

Lisowski, Józef

Liszkowska, Joanna

Liu, Chaoxing

Liu, Di

Liu, Hexu

Liu, Huolong

Liu, Jih-Shun

Liu, Jinfeng

Liu, Keng-Ku

Liu, Peng

Liu, Qili

Liu, Ren-Shiou

Liu, Tong

Liu, Weiping

Liu, Xiaobo

Liu, Ying

Liu, Yuanqing 
Liu, Yung-Chuan

Liu, Yu-Peng

Llaria, Alvaro

Llorens, Eugenio

Lluna, Amparo Gamero

Lo Giudice, Angelina

Lo, Kin Hing

Lo, Kwong Fai Andrew

Lo, Wei-Shuo

Locatelli, Marcello

Locatelli, Vittorio

Locci, Antonio Mario

Lofland, Sam

Loginova, Irina

Lognay, Georges

Logue, Brian A.

Lohman, Jeremy

Long, Nguyen Van Duc

Longhurst, Philip

Longo, Maria A.

Longwic, Rafał

Lopac, Nikola

Lopes, Helena

Lopes, Marlene

Lopez Fernandez, Eduardo Jose

Lopez Martinez, Maria José

Lopez, Gartzen

López, J. Javier

Lopez, Manuel J.

López-Linares, Juan Carlos

López-Lorente, Ángela Inmaculada

López-Ochoa, Luis María

Loppi, Stefano

Łopusiewicz, Łukasz

Lorenz, Michael

Lorenzo, Miguel

Losch, Pit

Losurdo, Giuseppe

Louhenkilpi, Seppo

Low, Nicholas

$\mathrm{Lu}$, Chien-Yu

Lu, Chun-Ping

Lu, Huitian

$\mathrm{Lu}, \mathrm{Li}$

$\mathrm{Lu}$, Tao

Lu, Wei

Lu, Xingxu

$\mathrm{Lu}$, Xinyi

Łubiński, Jacek

Lucia, Umberto

Lúcio, Marlene
Lucke, Annegret

Ludwig, Roland

Ludwig, Wojciech

Luidold, Stefan

Luiza Pop, Izabela

Łukaszewicz, Andrzej

Lukaszewicz, Marcin

Lukitawesa, Lukitawesa

Luna Maldonado, Aurelio

Luna, Diego

Luo, Hao

Luo, Win-jet

Lupiáñez, José A.

Lusito, Letizia

Lyu, Pei

Lyu, Tao

M. Hangos, Katalin

Ma, Xiaoli

Ma, Yihong

Mac Dowell, Niall

Macak, Jan

Maccallini, Cristina

Macernis, Mindaugas

Machado, Idalina

Machicoane, Nathanael

Macia, Llorenc

Maciejczyk, Mateusz

Mackay, Robert

Madau, Fabio A.

Maddeboina, Krishnaiah

Madejski, Pawel

Maderuelo Martín, Cristina

Madhurapantula, Rama Sashank

Madleňák, Radovan

Madsen, Dag Øivind

Madzharov, Nikolay

Maertens, Luc

Maffei, Gianluca

Magalhães, Júlia M. C. S.

Magalhães, Leandro

Magalhães, Solange

Magdouli, Sara

Magi, Vinicio

Magierowski, Marcin

Magreñán, Ángel Alberto

Mahadik, Bhushan

Mahajan, Devinder

Mahapatra, Manoj K.

Mahar, Rohit

Mahboubi, Amir

Mahdinia, Ehsan 
Mahichi, Faezeh

Mahmud, Kishan

Mahmud, Md Readul

Maireles Torres, Pedro Jesus

Mais, Laura

Maj, Grzegorz

Majak, Jüri

Majdan, Marek

Majewska, Katarzyna

Majidi Nezhad, Meysam

Majka, Grzegorz

Majka, Jerzy

Majorana, Carmelo

Makareviciene, Violeta

Makeeva, Irina Mikhailovna

Makisha, Nikolay

Malachova, Katerina

Malakhov, Dmitri

Malavasi, Veronica

Malça, João

Malecha, Ziemowit

Malejko, Julita

Malfa, Giuseppe Antonio

Małgorzata, Kowalska

Malinowski, Marek T.

Malinowski, Mateusz

Malinowski, Szymon

Maňásková-Postlerová, Pavla

Manca, Maria L.

Mancinelli, Enrico

Mancini, Simone

Mandal, Abhirup

Mandal, Ronit

Mandal, Subhamoy

Manheim, Derek

Mania, Szymon

Manica, Rogerio

Manickam, Sivakumar

Manjunatha Reddy, Gollapalli Narayana

Mannheim, Viktoria

Manno, Roberta

Manno, Vincent

Mano, Joao F.

Manowska, Anna

Manso, Ricardo

Mansouri, Abraham

Manteca, Ángel

Mantione, Daniele

Manuel Vieira Pombo, António

Manuel, Pascual Guillamón

Manumachu, Ravi Reddy
Manzano, Hegoi

Manzo, Maurizio

Mao, Lei

Maraqa, Munjed A.

Marc, Gabriel

Marcello Falcone, Pasquale

Marchelli, Filippo

Marchi, Beatrice

Marchisio, Alberto

Marciniak-Lukasiak, Katarzyna

Marcinkowska, Agnieszka

Marcinkowski, Damian

Marco, Mauri

Marcu, Ioan Cezar

Marcucci, Giulio

Marek, Maciej

Marhavilas, Panagiotis

Marian, Max

Mariani, Alessandro

Mariani, Antonio

Mariasiu, Florin

Marichev, Kostiantyn

Mariello, Massimo

Marietta, Fodor

Marín, María Luisa

Marinca, Bogdan

Marinca, Vasile

Marinescu, Maria

Marini, Martino

Mariusz, Domagala

Mark, Ole

Markiewicz, Roksana

Markou, Giorgos

Markowicz-Piasecka, Magdalena

Marohnic, Tea

Marquez Ruiz, Alejandro

Marrasso, Elisa

Marrazzo, Pasquale

Marsano, Davide

Marshall, Nicholas

Marshall, Paul

Marsili, Enrico

Martegani, Enzo

Märtens, Olev

Martín, David Orellana

Martín, Domingo

Martin, Jessica

Martin, Richard

Martinaiou, Ioanna

Martínez, Sidonia

Martínez-Espinosa, Rosa María 
Martínez-Gómez, Pedro

Martín-Gil, Jesus

Martinka, Jozef

Martin-Rios, Carlos

Martins, Angela

Martins, Luisa

Martins, Luísa Margarida

Marto, Joana

Martone, Ivo

Martucci, Annalisa

Marucci, Alvaro

Marudova, Maria

Marusak, Piotr M.

Maruthamuthu, Murali

Marzantowicz, Łukasz

Marzec, Agata

Marzeddu, Simone

Marzocchella, Antonio

Masek, Vlastimil

Mashhadi, Peyman

Maskey, Mahesh Lal

Masłoń, Adam

Masood, Nahid Al

Masoudi Asil, Shima

Masoudi Soltani, Salman

Massa, Antonio

Mastanjević, Kristina

Masuda, Takako

Masullo, Mariorosario

Matas, Richard

Mateus, Pedro

Mathew, Shibin

Mathieu-Potvin, François

Mathivathanan, Logesh

Matijašić, Bojana Bogovič

Matijošius, Jonas

Matikainen, Marko

Matino, Ismael

Matos, Manuel

Matsuda, Keigo

Matsuda, Shinji

Matsumoto, Michiaki

Matwijczuk, Arkadiusz

Matykiewicz, Danuta

Mauk, Michael G.

Mauleón, Ignacio

Mavroidis, Ilias

Mavromatidis, Lazaros

Mayyahi, Ahmed Al

Mazat, Jean-Pierre

Mazinani, Saeed
Mazumder, Kishor

Mazurkiewicz, Dariusz

Mazurkiewicz, Jakub

Mazzei, Lorenzo

Mcauley, Kimberley

Mcreynolds, Katherine D.

Meagher, Robert

Mechrez, Guy

Meder, Roger

Medrano, Marc

Medvedev, Ilya Nikolaevich

Medyńska-Juraszek, Agnieszka

Meegan, Mary J

Mehli, Lisbeth

Mehrazma, Banafsheh

Mehta, Sunali

Mei, Hanfei

Meidute-Kavaliauskiene, Ieva

Meier, Robert J.

Meirelles De Souza, Mateus Nogueira

Mejuto, Juan

Meloni, Carlo

Meloni, Eugenio

Meloni, Stefano

Melucci, Dora

Memon, Tosifa

Mendes, Adélio

Mendiola, José

Mendonca, Fabio

Mendoza, Javier

Meneghetti, Antonella

Meneguzzo, Francesco

Menéndez, Miguel

Meneses, Lisandra

Meng, Xinghua

Menghini, Luigi

Menziani, Maria Cristina

Mercader, Pedro

Mercier-Bion, Florence

Merie, Violeta Valentina

Mesaros, Clementina A.

Meškys, Rolandas

Messina, Concetta

Messina, Giuseppe

Messineo, Antonio

Messyasz, Beata

Metcalfe, Benjamin

Metri, Prashant G.

Meyer-Kohlstock, Daniel

Miądlicki, Karol

Miao, Yu 
Miastkowska, Malgorzata

Miastkowska, Małgorzata

Micci, Michael

Michalcová, Vladimíra

Michalska, Anna

Michalski, Jacek A.

Michel, Carlos R.

MICHEL, Jean-Bernard

Michna, Aneta

Micic, Miodrag

Miciuła, Ireneusz

Midani, Mohamad

Miękus, Natalia

Migita, Satoshi

Migliori, Carmela Anna

Mikalauskienè, Asta

Mikhaylin, Sergey

Miki, Norihisa

Mikielewicz, Dariusz

Mikkelsen, Ove

Mikó, Balázs

Mikołajczyk, Tomasz

Mikulski, Dawid

Milanesio, Marco

Milán-García, Juan

Milazzo, Maria Francesca

Milčius, Darius

Milde, Moritz

Milichovský, František

Milioto, Stefana

Milković, Lidija

Millar, Michael-Allan

Miller, Gregory

Miller, Jason Rafe

Miller, Wolfram

Miloš, Müller

Milosan, Ioan

Minami, Ichiro

Minjares-Fuentes, Jose Rafael

Mio, Hiroshi

Miras, Haralampos N.

Mirás-Avalos, José Manuel

Mirek, Paweł

Miricioiu, Marius

Mironescu, Ion

Mironescu, Monica

Mironova, Maria

Miroslaw, Barbara

Mirzania, Pegah

Mishra, Anand

Mishra, Dhanada Kanta
Mishra, Kunal

Mishra, Nigam

Misiulia, Dzmitry

Mitra, Subhasish

Mitran, Gheorghița

Mittal, Nitesh

Mittelman, Brigit

Miyafuji, Hisashi

Miyawaki, Osato

Mizielińska, Małgorzata

Mladěnka, Přemysl

Mlejnková, Hana

Mocanu, Dan-Adrian

Mocek, Jan

Modenutti, Carlos

Modigell, Michael

Moeini, Seyed Sepehr

Mohammadi, Fazel

Mohammadian, Majid

Mohammed, Hussein A.

Mohammed, Iman

Mohammed, Nishil

Mohanram, Harini

Mohd-Yasin, Faisal

Mohebbi, Abolfazl

Moisan, Marie-Pierre

Moita, Ana Sofia

Moldovan, Macedon

Mole, Nikolaj

Moles, Norman

Molga, Eugeniusz

Molina-Torres, María-Pilar

Molino, Paul

Molinuevo-Salces, Beatriz

Molla, Mamun

Moller, James

Mombelli, Davide

Momenzadeh, Leila

Momete, Daniela

Momin, Mohammad Abdul Motalib

Mon, Yi-Jen

Mondal, Kunal

Mondal, Sudeepta

Monica, Costea

Moniello, Giuseppe

Moniz, Samuel

Monsuez, Jean-Jacques

Montalvo, Carlos

Montecchio, Daniele

Monteduro, Anna Grazia

Monteil-Rivera, Fanny 
Monteiro, Bernardo

Monteiro, Carlos

Monteiro, Eliseu

Montes, Antonio

Montes-García, Verónica

Montessori, Andrea

Montevecchi, Giuseppe

Montone, Carmela Maria

Montoya, Oscar Danilo

Monzón, Mario

Moo, James

Moore, Stuart

Moraga, Javier

Morales, Gabriel

Morales-Vera, Rodrigo

Moratti, Stephen C.

Moreno Beltrán, Plácido

Moreno Nieto, Daniel

Moreno, Jovita

Moretti, Laura

Morettini, Micaela

Morgan, Alex J. L.

Morganti, Pierfrancesco

Morgunov, Igor

Morkoc, Hadis

Morla, Ricardo

Moro, Artur J.

Morosini, Attilio Fiorini

Morozov, Vitaly A.

Morozova, Sofia

Morrone, Biagio

Morselli, Davide

Mosca Angelucci, Domenica

Moss, Elica M.

Mosselhy, Dina

Mostafazadeh, Ali Khosravanipour

Mostarac, Petar

Mottes, Monica

Motyl, Przemysław

Mourão, P. A. M.

Moussaoui, Younes

Moyano-Mendez, Jose Ramon

Mozaffari, Saeed

Mozdzierz, Marcin

Mramor, Katarina

Mrlík, Miroslav

Mrozik, Agnieszka

$\mathrm{Mu}$, Ruipu

Mucalo, Michael R.

Muchembled, Jerome

Mücke, Manfred
Mudunuru, Maruti Kumar

Muelas, David

Mügge, Carolin

Mugnano, Martina

Mukhametzyanov, Timur A.

Mukherjee, Raj

Mukherjee, Sankha

Mukherjee, Srijib K

Mukherjee, Tuhin

Mukhin, Nikolay

Müller, Joachim

Müller, Julian Marius

Müller, Timo

Muniz, Juan

Muñoz-Cerón, E.

Muntean, Edward

Muntean, Sebastian

Muntimadugu, Eameema

Murad, Sohail

Muresan, Andruta

Muresan, Vlad

Murias, Marek

Murillo-Marrodán, Alberto

Murino, Teresa

Murmura, Maria Anna

Muro, Maider

Murtada, Khaled

Musielak, Grzegorz

Musser, Jordan

Musuc, Adina Magdalena

Muszyński, Siemowit

Muszyński, Tomasz

Muthiah, Geethanjali

Muthu Karuppan, Mohan Kumar

Mutlu, Özge

Mysliwiec, Tami

Mystkowski, Arkadiusz

Myszograj, Sylwia

$\mathrm{Na}$, Dokyun

Nabeel, Muhammad

Nabi, Saleh

Nabiałek, Marcin

Nadulski, Rafał

Nævdal, Geir

Nagaraju, Ganji Purnachandra

Nagarkar, Amit

Nagarkar, Amit A.

Nagatani, Naoki

Nagy, Endre

Nagy, László

Naik, Akshata 
Naik, Shivangi

Nakagawa, Tetsuya

Nakamura, Hiroyuki

Nakamura, Hitomi

Nakano, Katsuyuki

Nakayama, Yuushou

Nalbandian, Michael J.

Naldi, Maurizio

Nalepa, Grzegorz

Nallu, Sumitha

Namgung, Ho

Naoki, Miyazato

Naoto, Shimizu

Naplocha, Krzysztof

Narasimhulu, Chandrakala Aluganti

Narayanan, Barath

Narciso, Javier

Naščáková, Jana

Naseri, Emad

Nastasi, Benedetto

Nastro, Alessandro

Nataliya, Dilna

Nath, Arijit

Natsui, Shungo

Naushad, Mu

Navarrete-Munoz, Eva Maria

Navarro Flores, Emmanuel

Navarro, Pablo

Navarro-Azorín, José Miguel

Naya, Shin-ichi

Nazir, Aamer

Nazzaro, Filomena

Nde, Divine Bup

Neag, Emilia

Neagu, Adrian

Nee, Matthew J.

Negahdar, Leila

Negrea, Petru

Nejad, Ali Farokhi

Nemchinova, Nina

Németh, Áron

Németh, Márton

Nemudry, Alexander

Neri, Alessandra

Neubauer, Peter

Neufeld, Janis S.

Neumann, Holger

Neumayer, Sebastian

Neunert, Grazyna

Neut, Christel

Nevaranta, Niko
Neves, Adriana Cunha

Neves, Maria da Graça P. M. S.

Newman, Kimberly E.

Nezam, Iman

Ng, Denny K. S.

$\mathrm{Ng}$, Ding-Quan

$\mathrm{Ng}$, Lisa C.

Nguyen, Khoi Tan

Nguyen, Quang D.

Nguyen, Trieu

Nguyen, Tuan

$\mathrm{Ni}$, Junjun

Nicolae, Marilena

Nicolai, Marisa

Nicolini, Andrea

Niculescu, Rodica

Niculescu, Violeta

Nielsen, Birthe

Nielsen, Søren Nors

Nikolaidis, Alexandros K.

Nikolaos, Karathanasopoulos

Nikolaou, Michael

Nikończuk, Piotr

Nilsson, R. Henrik

Nima, Nikvand

Nin, Stefania

Ninikas, Konstantinos

Niro, Serena

Nita, Irina

Niu, Ran

Nkomo, Simbarashe

Nobile, Stefano

Noble, Michael

Nocchetti, Morena

Noël, Sébastien

Nogueira, Idelfonso

Nogueira, Teresa

Nonappa, Nonappa

Noor, Nuruzzaman

Noppe, Eric

Norouzi, Omid

Noszczyńska, Magdalena

Nourian, A.

Novo, Luís

Nowacka, Małgorzata

Nowaczyk, Jacek

Nowak, Dariusz

Nowak, Dorota

Nowak, Krzysztof

Nowak-Woźny, Dorota

Nowicki, Michał 
Nsooudi, Nasim Nsooudi

Ntaikou, Ioanna

Ntatsi, Georgia

Ntavou, Erika

Ntougias, Spyridon

Nuez, Ignacio

Nunes, Leonel

Nunes, Leonel Jorge Ribeiro

Nunna, Bharath Babu

Nurmesniemi, Emma-Tuulia

Nyarko, Eric

Nzila, Alexis

Oancea, Gheorghe

Obrosov, Aleksei

Ochmian, Ireneusz

Ochodek, Tadeáš

Ochowiak, Marek

Oettingen, Mikolaj

Ogilvie, Sean

Oginni, Oluwatosin

Ogórek, Rafał

Ogunjirin, Adebowale

Ogut, Mehmet

Oh, Kwangseok

Ohba, Seigo

Ohenoja, Markku

Ohlsson, Jonas A.

Ohmura, Ryo

Ohto, Keisuke

Oikonomopoulos, Ioannis

Ojo, Ebenezer

Ojo, Peter

Okada, Yoshiharu

Okamoto, Satoru

Okamoto, Takashi

Okechukwu, Okorie

Okolo, Patrick Nwosa

Okonkwo, Christopher

Okosun, Tyamo

Oksiuta, Zbigniew

Olariu, Marius-Andrei

Olaru, Dumitru

Olba-Zięty, Ewelina

Olczak, Piotr

Olejniczak, Teresa

Olfs, Hans-Werner

Oliva, Sabrina

Oliveira Panão, Miguel R.

Oliveira Santos, Lino De

Oliveira, Hugo M.

Oliveira, Rúben
Olšiak, Róbert

Olszewska, Monika A

Olszewski, Robert

Olvera-Vargas, Hugo

Omar, Ahmad

Omasa, Takeshi

Ombres, Luciano

Omer, Siddig Adam

Onchoke, Kefa

Oniga, Ovidiu

Onishi, Viviani

Onoda, Hiroshi

Onofri, Fabrice R. A.

Onstein, Erling

Onwude, Daniel I.

Opoka, Wlodzimierz

Oprean, Radu

Oramas-Royo, Sandra

Orazbayev, Batyr

Orellana-Palma, Patricio

Orfanidis, Sotiris

Orian, Laura

Orkusz, Agnieszka

Ortiz, Carlos

Orynycz, Olga

Orzan, Gheorghe

Osakada, Kohtaro

Osara, Jude

Oshima, Yusuke

Oskay, Riza Görkem

Osman, Haitham

Ossowicz, Paula

Ostacolo, Carmine

Ostapiuk, Monika

Osterroth, Sebastian

Ostrowska-Ligęza, Ewa

Otremba, Zbigniew

Otsuki, Akira

Otto, Tauno

Ottogalli, Kiara

Otwinowski, Henryk

Ouahbi, Tariq

Ourliac-Garnier, Isabelle

Owen, Guillermo

Pacana, Andrzej

Paciulli, Maria

Pacławski, Adam

Păcularu-Burada, Bogdan

Padhye, Nikhil

Padilla-Rascón, Carmen

Padilla-Rivera, Alejandro 
Padnya, Pavel

Padovano, Antonio

Padrão, Jorge

Padrosa, David

Paeng, Kijung

Pagano, Cinzia

Pagnacco, Maja C.

Pahl, Julia

Paiva, Alexandre

Paixão Cansado, Isabel Pestana

Pakdel, Esfandiar

Pal, Nabamita

Paladino, Ombretta

Palazzo, Ida

Palczynska, Beata

Paleo Vieito, António Jose

Palma, Carolina Font

Palmieri, Ferdinando

Palo, Emma

Palomba, Valeria

Paluch, Andrew S.

Paluch, Emil

Palumbo, Andrea

Palumbo, Domenico

Palumbo, Oriele

Pamuković, Jelena Kilić

Pan, Jeng-Shyang

Pan, Wei-Ping

Panaro, Maria Antonietta

Panas, Andrzej

Panderi, Irene

Pandyaswargo, Andante Hadi

Panek, Rafal

Panepinto, Deborah

Pang, Bo

Pang, Cheng-Yoong

Pang, Shuo

Pang, Shusheng

Panico, Antonio

Pannkuk, Evan

Panos, Anthony L.

Panzarasa, Guido

Paolella, Andrea

Paolone, Annalisa

Paone, Emilia

Pap, Bálint

Papa, Federico

Papadakis, Andreas

Papadopoulos, Basil

Papahristou, Evridiki

Papakyriakou, Athanasios
Papale, Maria

Papangelo, Antonio

Papapolymeroua, Georgios A. P.

Papathanasiou, Thanasis D.

Papenberg, Nikolaus

Papi, Alessio

Parajuli, Durga

Paralič, Ján

Paramythiotou, Elizabeth

Parchami, Mohsen

Pardo Picazo, Miguel Ángel

Parian, Mehdi

Parida, Dambarudhar

Parikesit, Arli Aditya

Paris, Enrico

Park, Choon-Su

Park, Chul-ho

Park, Donghee

Park, Eun Duck

Park, Herie

Park, Ilhwan

Park, Inhwan

Park, Jaehyun

Park, Jang-Hyun

Park, Jay Hoon

Park, Jin Yong

Park, Joontaek

Park, Jungsu

Park, Kang Hyun

Park, Kiho

Park, Min Bum

Park, Myung-June

Park, Nokuk

Park, Soo Jean

Park, Sunyoung

Park, Woo Jae

Park, Young-Tae

Parlinska-Wojtan, Magdalena

Parnell, Steven

Parnian, Mohammad Javad

Parra Boronat, Lorena

Parrondo-Gayo, Jorge Luis

Parsa, Shima

Parsons, Jason

Partoon, Behzad

Parys, Wioletta

Parzentny, Henryk R.

Pasculli, Antonio

Pashikanti, Srinath

Pašić, Borivoje

Passaglia, Elisa 
Pasti, Luisa

Pastor, Isidro M.

Patalas-Maliszewska, Justyna

Patanè, Salvatore

Patel, Rajnikant

Patelski, Piotr

Pater, Sebastian

Paterson, Nigel

Pati, Dipa

PATIL, SHRADHA

Patil, Vikram

Patra, Hirak K.

Patriarca, Riccardo

Patterson, Albert E.

Paul, Narayan Chandra

Paul, Shuva

Paul, Titan

Paul, Titan C.

Paulauskaite-Taraseviciene, Agne

Paulauskiene, Tatjana

Pau-Roblot, Corinne

Pavagadhi, Shruti

Pavaloaia, Vasile Daniel

Pavan, Paolo

Pavlenko, Ivan

Pavliňáková, Veronika

Pavloudi, Christina

Pavlyuchenko, Evgeniy

Pawar, Shrikant D.

Pawar, Sudhanshu

Pawełczyk, Anna

Pawlak, Maciej

Pawłat, Joanna

Pawłowska, Agnieszka

Pawlowski, Andrzej

Pawlowski, Lucjan

Paz Hernandez, Rubén

Paz-Ferreiro, Jorge

Pecht, Michael Gerard

Peeters, Floran

Pei, Yan

Pellegrini, Marco

Pellegrini, Riccardo

Pellegrino, Roberta

Pelton, Arthur

Peña Fernández, María Ángeles

Peña-Gómez, Nataly

Peña-Martínez, Ramón

Peñas, Pablo

Pencheva, Tania

Peng, Robert $\mathrm{Y}$.
Penkova, Nina

Penlidis, Alexander

Penn, Gregory

Pennetta, Antonio

Pennisi, Luca

Pennone, Vincenzo

Penumarti, Anusha

Pepi, Milva

Perales, A.L. Villanueva

Perche, Federico

Perdoch, Waldemar

Perec, Andrzej

Pereira, Leonel

Pereira, Olívia R.

Peres, José

Perestrelo, Rosa Maria De Sá

Perez De Souza, Leonardo

Perez Del Real, Rafael

Pérez Fernández, Raúl

Pérez, Alberto T.

Perez, Felipe

Perez-Gavilan, Ariane

Pérez-Monserrat, Elena Mercedes

Pérez-Sánchez, Modesto

Pericleous, Koulis

Perkumienė, Dalia

Perna, Filippo

Peron, Gregorio

Persson, Kenneth M.

Perucka, Irena

Pescaru, Dan

Petcu, Dana

Petelska, Aneta

Peter Nadeem, Simon

Peter, Barkoczy

Peter, Francisc

Peterson, Eric

Peterson, Larryn

Petračić, Ana

Petrashova, Dina

Petrásková, Lucie

Petravić, Janka

Petriev, Iliya

Petrillo, Antonella

Petrou, Christos

Petrovski, Željko

Petrusevski, Branislav

Petrzela, Jiri

Pettersen, Johan Berg

Peyman, Razi

Pfost, Heiner 
Pham, Anh Tung

Pham, Phuong

Phuntsho, Sherub

Piatek, Pawel

Piątkowski, Marcin

Piątkowski, Marek

Piccarozzi, Michela

Picchio, Rodolfo

Picó Vila, Rubén

Pico, Jesus

Piegat, Agnieszka

Piekoszewski, Wojciech

Pielech-Przybylska, Katarzyna

Pierini, Filippo

Pieta, Izabela S.

Pietraszek, Jacek

Pietrogrande, Maria Chiara

Pietrowicz, Slawomir

Pietrzyk, Stanislaw

Pietrzyk, Stanisław

Pietrzyk-Brzezinska, Agnieszka J.

Piga, Luigi

Pilarska, Agnieszka

Pilarska, Agnieszka A.

Piłatowska, Mariola

Pilicheva, Bissera

Pimienta, Rodney Lacret

Pineault, Nicolas

Pinela, José

Pingitore, Nicholas

Pinho, Henrique J.O.

Pino, Lidia

Pinto, Gustavo Filipe

Pinto, Loris

Piot-Lepetit, Isabelle

Piotr, Lichota

Piotrowski, Paweł

Piotrowski, Robert

Pipintakos, Georgios

Pipiška, Martin

Piqueras, Pedro

Piracha, Afaq

Pirola, Yuri

Pirooznia, Mehdi

Pisano, Roberto

Pisaturo, Giuseppe Roberto

Piscopo, Vincenzo

Piska, Kamil

Piskur, Paweł

Píštěk, Václav

Pitas, Charalampos N.
Piwowarski, Marian

Piyasena, Menake

Pizzi, Antonio

Plastina, Pierluigi

Plevris, Vagelis

Ploskas, Nikolaos

Plotka, Magdalena

Plymale, Andrew

Poch, Manel

Pocol, Cristina Bianca

Poddelsky, Andrey

Podgursky, Vitali

Pohar, Andrej

Pohjankukka, Jonne

Pohl, Pawel

Poiana, Marco

Polenghi, Adalberto

Poletto, Massimo

Polinski, Jaroslaw

Polivtseva, Svetlana

Poljak, Igor

Polverino, Pierpaolo

Pomi, Raffaella

Poniewiera, Marian

Poniewski, Mieczysław

Pons, Josefina

Pontrelli, Sammy

Pop, Ioan

Pop, Mihaela

POPESCU, Dumitru

Popescu, Ionel Catalin

Poponi, Stefano

Popovic-Gerber, Jelena

Popovich, David

Popp, József

Portugal, Camila C.

Porubská, Mária

Posel, Zbyšek

Poskrobko, Sławomir

Poulin, Francis J.

Pouliot, Yves

Poupot, Remy

Pouquet, Annick

Pourhashem, Ghasideh

Pous Rodríguez, Narcís

Pradhan, Kallol

Prager, Maximilian

Pragliola, Stefania

Praks, Pavel

Praplan, Arnaud P.

Prashar, Sanjiv 
Pratt, Lawrence

Prete, Roberta

Preux, Christophe

Prieto, Jose

Procentese, Alessandra

Prodan, Maria

Proença Brójo, Francisco Miguel Ribeiro

Prokop, Ales

Prokopov, Tsvetko

Prończuk, Mateusz

Proto, Andrea

Prozzi, Marco

Przybyłek, Maciej

Przystupa, Krzysztof

Psomopoulos, Constantinos S.

Ptak, Szymon

Puccioni, Sergio

Puchlik, Monika

Pueppke, Steven

Pugna, Irina Bogdana

Pujol-Nadal, Ramón

Pukalskas, Saugirdas

Pullman, David P.

Puna, Jaime

Puscaselu, Roxana

Putta, Koteswara Rao

Puttinger, Stefan

Pyataev, Nikolay A.

Pycia, Karolina

Pyda, Waldemar

Pyka-Pająk, Alina

Pylypchuk, Ievgen V.

Pypłacz, Paula

Pyzik, Adam

Qi, Fenglei

Qi, Zheng

Qian, Xuejun

Qiao, $\mathrm{Yu}$

Qin, Lang

Qin, Wensheng

Qin, Yi

Qu, Chuang

Quadrelli, Paolo

Quantin, Cécile

Quaranta, Emanuele

Quaranta, Giovanni

Queiros, Carla

Quero, Amparo Jimenez

Quero, David

Quesada, David

Quesada, Isabel Fernández
Quinson, Jonathan

Raayai, Shabnam

Rabissi, Claudio

Race, Marco

Race, Paul

Rachel, Hoo Poh Ying

Racoviceanu (Babuta), Roxana

Radac, Mircea-Bogdan

Radhakrishnan, T. K.

Radko, Lidia

Radtke, Aleksandra

Radulescu, Corina

Radulovic, Jovana

Radziemska, Maja

Rafalskaya, Tatyana

Raffelt, Klaus

Rafieenia, Razieh

Raganati, Federica

Raganati, Francesca

Rahimi-Gorji, Mohammad

Rahimzadeh, Iman

Rahman, Arifur

Rahman, Ashfaqur

Rahman, Ashiqur X.

Rahman, Md Mofijur

Rahman, Md Motiur

Rahman, Moksadur

Rahman, Muhommad Azizur

Rahmati-Abkenar, Mahboubeh

Rahnama, Mostafa

Rajamani, Raj K.

Rajda, Janusz

Rajska, Maria

Rajsl, Ivan

Raju, Arun Satheesh Kumar

Rakhmangulov, Aleksandr

Rakoczy, Rafał

Raman, Senthil Kumar

Ramantas, Kostas

Ramasamy, Elamparuthi

Ramírez-Argáez, Marco

Ramón Jerónimo, Juan Manuel

Ramon, Jan

Ramos, Ana

Ramos, Helena M.

Ramos, Sérgio

Ramos-López, Darío

Rana, Ziaul Hasan

Ranacher, Lea

Ranade, Vivek V.

Rantuch, Peter 
Rao, Govind

Rapa, Mattia

Raposo, Maria

Raposo, Maria Manuela

Rarita, Luigi

Rasoulianboroujeni, Morteza

Rastija, Vesna

Rathinam, Navanietha Krishnaraj

Ratnaweera, Harsha

Ratti, Stefano

Rauh, Andreas

Raush, Gustavo

Ravula, Sudhir

Rayguru, Madan Mohan

Razboršek, Maša Islamčević

Rebaudengo, Manuela

Recek, Nina

Reczek, Lidia

Reddy, M. V.

Reddy, Sohail R.

Redlarski, Grzegorz

Redondo-Foj, Belen

Regmi, Samundra

Rehrl, Jakob

Reible, Danny

Reid, Russell C.

Reincke, Katrin

Reinikainen, Matti

Reis, Anabela

Reis, Catarina Pinto

Rejdak, Michał

Relich, Marcin

Rella, Roberto

Remedios Serrano, Dolores

Remigante, Alessia

Remmas, Nikolaos

Ren, Shisong

Rene, Eldon

Renella, Giancarlo

Renz, Michael

Renzi, Massimiliano

Repajić, Maja

Restaino, Odile Francesca

Revnic, Cornelia

Revollar, Silvana

Reyes Belmonte, Miguel Ángel

Reyes Menéndez, Ana

Reynisson, Jóhannes

Reza, M. Toufiq

Rezaei Gomari, Sina

Rezapour Mashhadi, Mohammad Mahdi
Rezek Jambrak, Anet

Rezg, Nidhal

Rezk, Ahmed

Rezvani, Mohsen

Rhi, Seok-Ho

Rhinehart, R. Russell

Riazi, Hossein

Ribechini, Erika

Ribeiro, Thierry

Riccardi, Claudia

Ricci, Arianna

Ricci, Marilena

Riccò, Raffaele

Richards, Nia

Richards, Tobias

Richter, Andreas

Riela, Serena

Riese, Julia

Rigalli, Juan Pablo

Righi, Marco

Rigoni, Federica

Rikmann, Ergo

Rimac, Hrvoje

Rimkus, Alfredas

Rincon, David

Rincón, Esther

Ritos, Konstantinos

Rivas, Alejandro

RIvera, Rodolfo Marin

Rizun, Nina

Rizvi, Syed Husain Mustafa

Rizzi, Vito

Rizzuti, Bruno

Robertovna, Garsiya Ekaterina

Roberts, Janet

Robles, Pedro

Robles-Rodriguez, Carlos E.

Robusto, Francesco

Rocchetti, Gabriele

Rocchitta, Gaia

Rocha, Francisco

Rocha, Hugo

Roco, José Miguel Mateos

Rodger, James

Rodrigue, Denis

Rodrigues, Eduardo M. G.

Rodrigues, Francisca

Rodrigues, Márcio

Rodríguez De Rivera, Óscar

Rodriguez Rojo, Soraya

Rodríguez, Camino Fernández 
Rodríguez, Javier

Rodriguez-Couto, Susana

Rodríguez-Monroy, Carlos

Rodríguez-Solana, Raquel

Rodziewicz, Joanna

Roekaerts, Dirk J. E. M.

Rogala, Andrzej

Rogala, Zbigniew

Rogié, Brice

Rogovoy, Anatoly A.

Rogowski, Krzysztof

Roh, Changhyun

Rokosz, Krzysztof

Roman, Andres

Roman, Monika

Román, Silvia

Romano, Elio

Romański, Jarosław

Romar, Henrik

Romero, Inmaculada

Romuli, Sebastian

Roohnikan, Mahdi

Ropital, François

Roppolo, Ignazio

Rosa, Paolo

Rosa, Pedro

Rosa, Tundis

Rosaria, Sciarrillo

Rosenthal, Katrin

Rosentrater, Kurt A.

Roskosz, Maciej

Rossetti, Ilenia

Rossi, Fabrizio

Rossi, Franca

Rossiter, Anthony

Rotaru, Aurelian

Roubík, Hynek

Rousseau, Guy

Roussis, Ioannis G.

Rout, Bapin Kumar

Routray, Winny

Row, Kyung Ho

Roy Choudhury, Shreya

Roy, Dipankar

Roy, Partho Sarothi

Roy, Sukanta

Roycroft, Brendan

Royer, Jean-Jacques

Rozbroj, Jiří

Rozwadowski, Tomasz

Różyło, Renata
Ruban, Dmitry

Rubiano, Jesús García

Rubilotta, Emanuele

Rubino, Edoardo

Rubio, Juan Luis

Rudolf, Pavel

Rudra, Souman

Rudzinska, Magdalena

Ruffin, Paul

Ruffino, Barbara

Ruffo, Francesco

Ruiz Larrea, Fernanda

Ruiz-García, Alejandro

Ruiz-Rosas, Ramiro Rafael

Rusinek, Robert

Rusowicz, Artur

Russo, Francesco

Russo, Maria Elena

Russo, Mario Vincenzo

Russo, Pasquale

Russo, Vincenzo

Rusu, Daniela

Ruta, Lavinia

Ruthstein, Sharon

Rutkowska, Jaroslawa

Rutkowska-Zbik, Dorota

Ruuska, Jari

Ruysschaert, Jean Marie

Ryadnov, Max

Rybacki, Piotr

Rybak, Aleksandra

Rybarczyk, Piotr

Rydén, Magnus

Rydz, Dariusz

Rykowska, Iwona

Ryu, Jaiyoung

Ryu, Keun Ho

Ryu, Seo-Yoon

Rzelewska-Piekut, Martyna

Sa, Jeong-Hoon

Sabila, Paul

Sabirov, Denis

Saccani, Cesare

Sadeghifar, Hasan

Sadhasivam, Sudharsan

Sadowska, Anna

Sadraei, Razieh

Sáenz De Argandoña, Eneko

Sáez Bastante, Javier

Safaei, M. R.

Safdari Shadloo, Mostafa 
Safferman, Steven

Sager, Manfred

Saghir, Ziad

Sah, HongKee

Sah, Injin

Saharan, Lalita

Sahoo, Soumya R.

Sahroni, Taufik Roni

Sahu, Umakant

Saikova, Svetlana

Saini, Rahul

Saini, Ramesh Kumar

Saini, Sharanjot

Saito, Mari M.

Sajavičius, Svajūnas

Sajdak, Marcin

Sajid, Muhammad

Sakai, Osamu

Sakuragi, Kiyoshi

Saladino, Maria Luisa

Salahub, Dennis

Salar-Garcia, M. J.

Salas, Santiago

Saleem, Arslan

Salehi, Maryam

Salehi, Saeed

Salehzadeh-Yazdi, Ali

Salerno, Carlo

Salgansky, Eugene Aleksandrovichn

Šalić, Anita

Saliga, Jan

Salimi, Mehdi

Salman, Chaudhary Awais

Salmas, Constantinos

Salmeia, Khalifah A.

Salmon, Morgan

Salvadori, Simone

Salvestrini, Stefano

Samanidou, Victoria

Samaniuk, Joseph R.

Sambucci, Matteo

Samhaber, W. M.

Samková, Eva

Sanchez Lasheras, Fernando

Sanchez Muñoz, Raul

Sánchez, Carlos

Sanchez, Fabio A. Cruz

Sanchez-Romero, Jose-Luis

Sánchez-Vázquez, Rebeca

Sanchis-Sebastiá, Miguel

Sander, Aleksandra
Sandín-España, Pilar

Sanna, Gavino

Sannino, Filomena

Sansone, Anna

Santamaria, Monica

Santangelo, Paolo E.

Santhanam, Kalathur

Santi, Melissa

Santolaria Morros, Carlos

Santolini, Enrica

Santos De Gois, Jefferson

Santos Juanes-Jorda, Lucas

Santos, Carla S.

Santos, Catarina

Santos, Rafael

Santos, Valentín

San-Valero, Pau

Sanvito, Francesco Davide

Sanz-Calcedo, Justo Garcia

Sapudom, Jiranuwat

Sara, Zalba

Sarabia, Aida Maria Diez

Saravanakumar, Kandasamy

Sarma, Pranjal

Sarma, Saurav

Sarmad, Shokat

Sarna, Michal

Sarraguça, Mafalda

Sasmal, Aniruddha

Sassi, Mohamed

Satoh, Hisashi

Saturnino, Carmela

Savkovic, Borislav

Sawik, Tadeusz

Sayegh, Marderos

Sayydi, Nima

Sazonova, Margarita A.

Sbrizzai, Roberto

Scarano, Antonio

Scargiali, Francesca

Scarpa, Marina

Schiffner, Ingo

Schiraldi, Chiara

Schleich, Benjamin

Schlesinger, Mark

Schmidt, Bernhard V .K. J.

Schmidt, Jochen

Schmidt-Bleek, Katharina

Schmitz, Nico

Schneider, Daniel Rolph

Schneider, Marius 
Schneider, Raphael

Scholz, Miklas

Schreiber, Anja

Schreiber, Igor

Schröder, Agnes

Schubert, Peter J.

Schuck, Alyssa

Schulte, Horst

Schulz, Volker Paul

Schumacher, Sandra

Schuurman, Michiel J.

Schwaminger, Sebastian

Schwarz, Ulrich

Schwarzkopf, Julia

Seca, Ana Maria Loureiro Da

Sedláková, Jana

Seelam, Prem Kumar

Seet, Gerald Gim Lee

Segal, Rebecca

Segec, Pavel

Seijo, María Carmen

Seiler, Philipp

Seiner, Brienne N.

Seitz, Jochen

Sęk, Jerzy

Selin, Victor

Selke, Matthias

Selosse, Sandrine

Sementa, Luca

Semkov, Krum

Sempere, Ignacio Ruigómez

Senadeera, Wijitha

Sendžikienè, Eglè

Sene, Ndolane

Senila, Lacrimioara

Sepelevs, Igor

Sepulveda-Escribano, Antonio

Sequeira, César Augusto Correia De

Serani, Andrea

Sereikaite, Jolanta

Serna-Loaiza, Sebastián

Serrà, Albert

Serrano, Clara

Serrano-Aroca, Ángel

Seruga, Przemysław

Sevilimedu Veeravalli, Sathyanarayanan

Seyfang, Bernhard

Shadloo, Mostafa Safdari

Shafagh, Ida

Shafer, Wilson

Shafiei, Ali
Shahzad, Muhammad Wakil

Shakhtshneider, Tatyana P.

Shakouri, Abolfazl

Shala, Ahmet

Shang, Jin Eric

Shang, Qingsen

Shang, Yilun

Shankar, Eswar

Shankarappa, Sahadev A.

Shankles, Peter

Shao, Jiankun

Shao, Shuai

Sharan, Nek

Shariare, Mohammad

Sharifi, Amin

Sharker, Shazid Md.

Sharma, Kavita

Shen, Dongyuan

Shen, Jialong

Shenderovich, Ilya G.

Sheng, Ruilong

Shengo, Michel

Shepa, Ivan

Sher, Farooq

Sherbakov, Sergei

Sherrell, Peter

Shevchuk, Igor

Shi, Jingyi

Shi, Xiao-Lei

Shi, Xiaoyang

Shi, Zhenguo

Shiau, Lieding

Shieh, Hsin-Jang

Shimizu, Kazuo

Shimizu, Kazuyuki

Shin, Dong Joo

Shin, Donghyuk

Shin, Hyun-Jae

Shin, Keesam

Shin, KwangSup

Shin, Minchul

Shin, Yoon Hyuk

Shinomiya, Kazufusa

Shirazinejad, Reza

Shishin, Denis

Shishkin, Andrei

Shitut, Shraddha

Shoda, Makoto

Shogenov, Kazbulat

Shojaei, Arman

Shoji, Yoshiaki 
Shokouhimehr, Mohammadreza

Shorstkii, Ivan

Short, Michael

Shrestha, Namita

Shrestha, Shikha

Shrirao, Anil

Shu, Chi-Min

Shu, Shuli

Shvidchenko, Aleksandr V.

Shvydka, Diana

Siatka, Tomáš

Sicari, Vincenzo

Sideratou, Zili

Sidorov, Denis Nikolai

Siegel, Stefan

Sieni, Elisabetta

Signore, Giovanni

Siikonen, Timo

Siipola, Virpi

Siirde, Andres

Sikora, Anna

Sikora, Karol

Sikorska, Ewa

Sili, Andrea

Silva, Amelia M.

Silva, Severiano R.

Sim, Hyung Sub

Simeonov, Ivan

Simha Martynkova, Grazyna

Simm, Stefan

Simon, Ulla

Simonato, Barbara

Simonič, Marjana

Simo-Tagne, Merlin

Simson, Alan

Sinama, Frantz

Sinche, María

Singh, Ashutoush

Singh, Gurwinder

Singh, Harpreet

SINGH, JASHBIR

Singh, Kamal Deep

Singh, Mehakpreet

Singh, Pradeep Kumar

Singh, Rekha

Singharoy, Abhishek

Sinha, Ashesh Kumar

Sinibaldi, Giorgia

Sipos, Emese

Širá, Elena

Siracusa, Rosalba
Siren, Heli

Sirugaloor, Senthilkumar

Sismanis, Panagiotis

Sitarek, Przemysław

Siuta, Dorota

Siwiec, Tadeusz

Skaldina, Oksana

Skaltsa, Liana

Skoczko, Iwona

Skórczewska, Katarzyna

Skordaris, Georgios

Skorska, Anna

Skouras, Eugene D.

Skruzny, Michal

Skrypnik, Liubov

Skrypnik, Liubov N.

Skrzypkowski, Krzysztof

Skylogianni, Eirini

Sloan, Jeremy

Slobodyan, Mikhail

Slomkiewicz, Piotr

Słomkiewicz, Piotr Marek

Ślusarczyk, Beata

Smith, Ellen M. Lavoie

Smith, Mark

Smith, Micholas Dean

Smith, Robert

Smith, Scott R.

Smoczyński, Lech

Smolensky, Dmitriy

Smoliński, Adam

Smyk, Emil

Soares, Cristina

Sobczak, Paweł

Sobeh, Mansour

Sobiecka, Elżbieta

Sobiesiak, Magdalena

Sobieski, Wojciech

Sobota, Tomasz

Socas, Rafael

Socas-Rodríguez, Bárbara

Soco, Eleonora

Soda, Josko

Sodano, Valeri

Sohn, Youngku

Șoimoșan, Teodora Melania

Solana, Raquel Rodríguez

Solano, Juan Pedro

Soldado, Rosana Montes

Soldatkina, Liudmyla

Soler, Jaime 
Solina, Vittorio

Solis-Bravo, Gregorio

Solyanikova, Inna

Sommer, Julia

Son, Seongmin

Son, Yang-Ju

Sonavane, Manoj

Song, Sanghoon

Song, Wen

Song, Young Chae

Song, Young-Chae

Song, Young-hak

Soni, Aswathi

Sonnino, Giorgio

Soon, Jong Jeong

Sordello, Fabrizio

Sorescu, Ana Alexandra

Soriano, Prof. Enrique

Sorin, Mikhail

Sorrenti, Vincenzo

Sorrentino, Giancarlo

Sosnowski, Marcin

Sou, Keitaro

Soualah, Ahcene

Soucemarianadin, Arthur

Soucy, Gervais

Souravlas, Stavros

Sousa, Ana Margarida

Sousa, Ana Margarida Luís De

Sousa, Angela

Sousa, Joaquim

Spadafora, Natasha Damiana

Spanedda, Maria

Španielová, Hana

Spano, Giuseppe

Spatari, Sabrina

Spelzini, Darío

Spencer, Adrian

Spiak, Zofia

Spigarelli, Renato

Špiláček, Michal

Spitas, Vasilios

Spur, Bernd W.

Spychaj, Tadeusz

Spychala, Marcin

Srinivasan, Mahesh

Srinivasan, Seshadhri

Srivedavyasasri, Radhakrishnan

Stacewicz-Sapuntzakis, Maria

Stadnichenko, Andrey

Stádník, Luděk
Stagg-Williams, Susan M.

Stanaszek-Tomal, Elżbieta

Stanclik, Michal

Stănescu, Nicolae-Doru

Staniak, Mariola

Staniszewska, Monika

Stankevich, Nataliya

Stapley, Andrew

Starek, Małgorzata

Stasiak-Różańska, Lidia

Stąsiek, Jan

Staszak, Maciej

Stathopoulos, Costas

Staubli, Thomas

Stavropoulos, Panagiotis

Stavroulakis, Georgios E.

Steele, Barry

Steenhuis, Tammo

Stefan, Simona Catalina

Stefana, Elena

Stefanik, Andrzej

Stefaniuk, Dawid

Stefanowicz-Hajduk, Justyna

Stegenta-Dąbrowska, Sylwia

Stehlíková, Beáta

Steinbach, David

Steinlechner, Stefan

Stelescu, Maria-Daniela

Stelmachowski, Paweł

Stencel, Marek

Stepien, Krzysztof

Steudler, Susanne

Stoddard, Shana

Stoica, Virgil

Stopa, Jerzy M.

Stopic, Srecko

Storti Gajani, Giancarlo

Storti, Giuseppe

Stozhko, Natalia

Straka, L'uboslav

Stratakis, Antonios

Straže, Aleš

Striugas, Nerijus

Strle, Drago

Strubelt, Henning

Struga, Marta

Strungaru, Stefan-Adrian

Strunk, Jennifer

Stuart, David T.

Stuchlik, Stanislav

Stückler, Martin 
Študentová, Hana

Studzinska-Sroka, Elzbieta

Stuparu, Adrian

Styles, Michael Thomas

Stylianakis, Minas

$\mathrm{Su}$, Lihong

$\mathrm{Su}$, Tsung-chow

$\mathrm{Su}, \mathrm{Wen}-\mathrm{Yu}$

$\mathrm{Su}$, Xiaojun

Subramani Paranthaman, Balasubramani

Subramanian, Parthasarathi

Subramoniam, Ramesh

Sudol, Miroslaw

Suea-Ngam, Akkapol

Sugahara, Takeshi

Sui, Ran

Sui, Zhang

Sujka, Monika

Šulc, Radek

Suleria, Hafiz

Sun, Guangyong

Sun, Li

Sun, Shichen

Sun, Wenguang

Sun, Xiuxuan

Sun, Yong

Sundermann, Andreas

Sundqvist, Jan-Olov

Sungthong, Rungroch

Sunny, S. M. Nahian Al

Sunseri, Francesco

Supponen, Outi

Suquet, Jordi

Surace, Rossella

Surup, Gerrit Ralf

Surženkov, Andrei

Susanto, Hadi

Susarrey-Arce, Arturo

Sutowska, Marzena

Suzuki, Tomo

Svensson, Elin

Svensson, Sarah

Swada, Jeff

Świątek, Łukasz

Swinarew, Andrzej

Symes, Mark

Syngouna, Vasiliki

Syrpas, Michail

Sysko-Romanczuk, Sylwia

Sytykiewicz, Hubert

Szabó, Balázs P.
Szaleniec, Maciej

Szamałek, Krzysztof

Számel, László

Szatyłowicz, Jan

Szczepanik, Beata

Szcześniak, Paweł

Szczucka-Lasota, Bożena

Sze, Eric Tung-Po

Szederkényi, Gábor

Szentannai, Pal

Sziebig, Gabor

Szilveszter, Szabolcs

Szlachta, Józef

Szmelter-Jarosz, Agnieszka

Szostak, Elżbieta

Sztanke, Małgorzata

Sztekler, Karol

Szulc, Karolina

Szulc, Przemyslaw

Szumny, Antoni

Szwaja, Stanislaw

Szwajca, Anna

Szwengiel, Artur

SZYBIŃSKI, BOGDAN

Szychowski, Dariusz

Szydłowska, Aleksandra

Szymanek, Mariusz

Szymanowska, Urszula

Szymańska, Magdalena

Szymczyk, Anna

Szymczyk, Piotr

Szymiczek, Małgorzata

Tabakaev, Roman B.

Taddei, Federica

Taeibi-Rahni, Mohamad

Tagami, Takayoshi

Tagirov, Marat

Taheri, Pooya

Tahershamsi, Leili

Taherzadeh, Mohammad

Tajti, Ádám

Takada, Tomoya

Takafuji, Makoto

Takayuki, Tsukui

Takó, Miklós

Takosogulu, Jakub Emanuel

Taler, Dawid

Talsma, Herre

Țălu, Ștefan

Talukder, Byomkesh

Tama, Bayu Adhi 
Tamarin, Ollivier

Tamašauskaitè-Tamašiūnaitè, Loreta

Tamburrano, Paolo

Tamus, Zoltán Ádám

Tan, Alex Yong Kwang

Tan, Hua

Tan, Kuang-Hsiung

Tan, Raymond G.

Tanabe, Yoo

Tanaka, Mikiya

Tănasă, Constantin

Tanase, Corneliu

Tańczyk, Marek

Taner, Tolga

Tang, Xin

Tanner, Eden

Tanner, Ralph

Tanner, Roger I.

Tantawi, Khalid

Tanveer, Sheik

Tapia, John

Tapia, Jose A.

Tappia, Elena

Taqieddin, Amir

Tarabanko, Nikolay

Tarabanko, Valery E.

Tarczewska, Aneta

Tarighaleslami, Amir Hossein

Tarutani, Naoki

Tasinato, Nicola

Tassinari, Francesco

Tateda, Masafumi

Tavakoli, Javad

Taxiarchou, Maria

Tayyab, Muhammad

Tebbani, Sihem

Teeri, Teemu

TEHRANI, KAMBIZ

Tejado, Inés

Teleszewski, Tomasz

Telukutla, Srinivasa Reddy

Tenace, Valerio

Tenorio-Alfonso, Adrián

Teo, Nicholas

Teodoriu, Catalin

Teodosiu, Carmen

Tepljakov, Aleksei

Tera, Melania

Terpou, Antonia

Terracciano, Daniela

Terrell, Evan
Terryn, Sanne

Tervahauta, Arja I.

Teschner, Tom-Robin

Tesfaye, Fiseha

Tetsushi, Matsuda

Texido Bartes, Robert

Teymour, Fouad

Thackray, Richard

Thapliyal, Vivek

Thibault, Jules

Thiebault, Thomas

Thirunavukarasu, Deepak

Thiruvengadam, Muthu

Thitsartarn, Warintorn

Thiyagarajan, Karthick

Thomas, Dilip

Thomas, Maciej

Thomas, Morgan L.

Thomas, Raymond

Thompson, Gregory J.

Tiainen, Jonna

Tian, Furong

Tian, Liang

Tic, Wilhelm Jan

Tien, Wei-Hsin

Tillenkamp, Frank

Time, Rune W

Timko, Michael

Timson, David J.

Tina, Giuseppe

Tirado, Diego F.

Tirelli, Nicola

Titova, Ekaterina S.

Tkadleckova, Marketa

Tlak Gajger, Ivana

Tobiszewski, Marek

Toet, Gijsbert

Toikka, Alexander

Tole, Ilda

Tolkou, Athanasia

Tolochko, Oleg

Tolstykh, Tatyana

Toma, Milan

Tomar, Anuradha

Tomášková, Hana

Tomczyk, Krzysztof

Tomé, Liliana C.

Tomków, Jacek

Tomovic, Mileta M.

Tomovska, Radmila

Tomoyoshi, Nishimura 
Tonacci, Alessandro

Tonelli, Domenica

Tonelli, Flavio

Tong, Van-Canh

Tong, Wenming

Tonni, Ingrid

Torabi, Mohammadamin

Torbaghan, Mehran Eskandari

Torii, Shuichi

Tornabene, Francesco

Toro, Roberta

Toro, Roberta G.

Torre, Maria Luisa

Torres Vaamonde, José Enrique

Torres, Cristiana

Torres, Miguel Garcia

Torres, Pedro M. B.

Tóth, András József

Tran, Le Vu

Tran, Quang Anh

Tränckner, Jens

Trebar, Mira

Treideris, Marius

Trelea, Cristian

Trematerra, Amelia

Trens, Philippe

Treutler, Kai

Tribe, Lorena

Trif, Monica

Trincone, Antonio

Trinh, Minh-Chien

Tripp, Carl P.

Trivedi, Chirag

Trmčić, Aljoša

Trögl, Josef

Trubetskaya, Anna

Trucillo, Paolo

Tryggvason, Gretar

Tsai, Chen-Chi

Tsai, Pi-Jen

Tsai, Wen-tien

Tsaltas, Dimitris

Tsao, Chung Chen

Tsimpanogiannis, Ioannis N.

Tsinarakis, George

Tsioras, Petros

Tsiropoulou, Eirini Eleni

Tskhovrebov, Alexander G.

Tso, Edwin

Tsolas, Ioannis

Tsotsas, Evangelos
Tsotsis, Theodore T.

Tsoupras, Alexandros

TSUJI, Daiki

Tsuji, Yutaka

Tsujimoto, Yoshinobu

Tsukahara, Takahiro

Tsurkan, Mikhail V.

Tsutsui, Waterloo

Tucki, Karol

Tudosie, Alexandru-Nicolae

Tuegeh, Maickel

Tufa, Ramato Ashu

Tufariello, Maria

Tůma, Karel

Tundys, Blanka

Tunes, Matheus A.

Tung, Tran Thanh

Tuomikoski, Sari

Tuovinen, Tero

Turcu, Antoniu

Turcu, Antoniu Claudiu

Turhanen, Petri

Turrini, Federica

Tusek, Ana Jurinjak

Tuśnio, Norbert

Tutak, Magdalena

Tutak, Wojciech

Tuti, Simonetta

Tyagi, Sonika

Tylewicz, Urszula

Tyliszczak, Bożena

Tymecki, Lukasz

Tynjala, Tero

Tyrologou, Pavlos

Tytła, Malwina

Tzeremes, Nickolaos G.

Tziourtzioumis, Dimitrios N.

Tziveleka, Leto-Aikaterini

Tzougas, George

Ubeda, Cristina

Uchida, Tsutomu

Uciński, Dariusz

Uddi, Mruthunjaya

Udert, Kai M.

Uetani, Kojiro

Ugrina, Marin

Ugwu, Johnson

Uhlenhut, Frank

Ujor, Victor C.

Ukrainets, Igor V.

Ul Haq, Rizwan 
Ulaganathan, Vamseekrishna

Ullah, AMM Sharif

Upadhyay, Mukesh

Urban, Wieslaw

Urbaniec, Krzysztof

Urbański, Mariusz

Usama, Syed Muhammad

Uscilowska, Anita

Ushida, Akiomi

Usman, Muhammad

Usuda, Haruki

Utsumi, Shigenori

Vadacca, Luigi

Vahid, Vajihinejad

Vaiana, Nicolò

Vaiano, Fabio

Vaikuntanathan, Visakh

Vaimann, Toomas

Vala, Jiri

Valcic, Marko

Valdés García, Arantzazu

Valdiserri, Paolo

Valeev, Dmitry

Valero, Daniel

Valero-Romero, María José

Valetti, Francesca

Valíček, Ján

Vallicelli, Elia Arturo

Valverde, Raul

Van Beek, Kees

Van Bockstaele, Filip

Van Dam, Pieter

Van Der Kamp, Jan Willem

Van Der Schaaf, Hylke

Van Duijsen, Peter

Van Dyk, Susan

Van Hal, Jaap

Van Lier, Ben

van Ommen, J. Ruud

Vannini, Andrea

Vannozzi, Lorenzo

Váradi, Csaba

Variny, Miroslav

Varnava, Kyriakos

Varner, Jeffrey

Varra, Michela

Vashurin, Artur

Vasková, Iveta

Vasudevan, Suraj

Vattepu, Ravi

Vaz, Josiana A.
Vazquez Espinosa, Mercedes

Vazquez, Francisco

Vecchio Ciprioti, Stefano

Vedagopuram, Sreekanth

Vega Reyes, Francisco

Veiga, Manuel Fernández

Velić, Natalija

Vella, Filomena Monica

Venegas, Maria

Venkatasubramanian, Anandram

Venskutonis, Petras Rimantas

Ventura, Cinzia Anna

Venus, Joachim

Venuti, Elisabetta

Vera García, Francisco

Vercelli, Barbara

Verdin, Patrick

Verdú-Andrés, Jorge

Vergara, José

Verma, Chaman

Verma, Puneet

Verma, Ranjit

Veronesi, Federico

Verros, George

Veskoukis, Aristidis S.

Vialetto, Giulio

Viapiana, Agnieszka

Vibhu, Vaibhav

Vibhute, Sandip M.

Vicente Crespo, Gemma

Vicente, Yolanda Sánchez

Victor, Vijay

Vidnerová, Petra

Vieira, Vasco M. N. C. S.

Viel, Marie

Vigier, Karine

Viglašová, Eva

Vijaya, Raghavan

Vijayavenkataraman, Sanjairaj

Vilardi, Giorgio

Vilas, José Luís

Vilke, Siniša

Villani, Maria Luisa

Villanneau, Richard

Vincenti, Sophie

Vincenzetti, Silvia

Vinci, Maria Cristina

Vinokurov, Vladimir

Virjamo, Virpi

Visconti, Paolo

Visuri, Ville-Valtteri 
Viswanathan, Harish

Viswanathan, Mothi Bharath

Vita, Antonio

Vitale, Gianpaolo

Vitale, Marina Consuelo

Vitale, Stefania

Vítěz, Tomaš

Vítěz, Tomáš

Vítězová, Monika

Vítor, Geraldes

Vítová, Milada

Vittorini, Pierpaolo

Viva, Federico A.

Viviani, Massimo

Vlachos, Nicholas

Vlădoiu, Rodica

Vlcak, Petr

Vo, Duc-Thang

Vo, Truong

Vochin, Marius

Vogel, Pierre

Vogt, Christian

Volcho, Konstantin

Volker, Körstgens

Volkov, Vladimir

Vollprecht, Daniel

Volosencu, Constantin

Volpe, Maurizio

Volpe, Rosaria

Volpi, Valentina

Von Bergen, Martin

Von Cossel, Moritz

Von Jouanne, Annette

Von Lieres, Eric

Vorauer-Uhl, Karola

Vorhauer, Nicole

Vorozhtsov, Alexander

Voskanyan, Albert

Voyiatzis, George

Vrabič Brodnjak, Urška

Vriesekoop, Frank

Vulin, Domagoj

Vuong, Thanh Huyen

Wada, Ken-Ichi

Wada, Toru

Wade, Matthew

Wagh, Priyesh

Wagh, Priyesh A.

Wahed, Arifeen

Wang, A-Cheng

Wang, Baicun
Wang, Ben

Wang, Can

Wang, Chao-Min

Wang, Chen

Wang, Chih-Feng

Wang, Chuan

Wang, Dian

Wang, Di-Yan

Wang, Endong

Wang, Fu-Kwun

Wang, Guichao

Wang, Hai

Wang, Heng

Wang, Huizhi

Wang, Jeffrey

Wang, Jingyu

Wang, Jinsheng

Wang, Junfeng

Wang, Kai

Wang, Kan

Wang, Leyu (Douglas)

Wang, Li-Fei

Wang, Lihua

Wang, Lu

Wang, Meng

Wang, Pei-Ming

Wang, Penghao

Wang, Pixiang

Wang, Qinyi

Wang, Shengnian

Wang, Shu

Wang, Shue

Wang, Tao

Wang, Tianhao

Wang, Weiqian

Wang, Xianzhi

Wang, Xiaoen

Wang, Xiaolin

Wang, Xiaoming

Wang, Xuewen

Wang, Yanchang

Wang, Yawen

Wang, Yu

Wang, Yun

Wang, Yun-Yan

Wang, Zhongyang

Wanner, Juergen

Warcholiński, Bogdan

Warchulski, Rafał

Ward, Alastair James

Warguła, Łukasz 
Wąs, Adam

Wasik, Michał

Wąsowski, Jacek

Watkins, James

Wawro, Aleksandra

Weber, Bart

Weber, Jürgen

Weber, Matthieu

Ween, Ola

Węglowski, Marek

Wei, Zongsu

Weide, Tobias

Weidinger, Tamás

Weigand, Wolfgang

Weiss, Clemens K.

Weiss, Leland

Wen, Xin

Weng, Fang-bor

Weng, Guo-Ming

Weng, Yung-Jin

Wenthold, Paul G.

Weres, Jerzy

Wesolowski, Krzysztof

West, Andrew

West, James D.

Weston, Javen

White, Alvin

White-Sanchez, Juan

Wiącek, Joanna

Widanagamage, Inoka $\mathrm{H}$.

Widera, Barbara

Wiedman, Gregory

Wiens, Travis

Wierzbicki, Jędrzej

Wietschel, Martin

Wiklund, Johan

Wiktor, Artur

Wildi, Walter

Wilk, Andrzej

Wilk, Klaudia

Wilkosz, Kazimierz

Williams, John A.

Williams, Kathryn R.

Wills, Ron

Wilpiszewska, Katarzyna

Winiczenko, Radoslaw

Winiczenko, Radosław

Wińska, Katarzyna

Wiraja, Christian

Wiśniewski, Tomasz

Wiszumirska, Karolina
Witaszek, Kamil

Witczak, Joanna

Witonska, Izabela

Witrick, Katherine

Witt, Katarzyna

Witz, Christian

Wiwatanapataphee, Benchawan

Wladyslaw, Mitianiec

Wlazło, Łukasz

Włoch, Marcin

Włodarczyk, Paweł P.

Włodarczyk, Renata

Włodarczyk-Stasiak, Marzena

Wohlrab, Sebastian

Wójciak, Magdalena

Wojciechowicz-Budzisz, Agata

Wojdyło, Aneta

Wojs, Marcin K.

Wojszczyk, Rafał

Wojtaszek, Małgorzata

Wójtowicz, Ryszard

Wojtusik, Mateusz

Wolff, Max

Wolny-Koładka, Katarzyna

Wołowicz, Anna

Wołowiec-Korecka, Emilia

Won, Jongmuk

Won, Seunggun

Won, Wangyun

Wondrak, Thomas

Wong, Bryan

Wong, Hsi-Wu

Wong, M. L. Dennis

Wong, Maurice

Wong, Voon-Loong

Woo, Kyeongsik

Woo, Sun Hee

Wood, Delilah

Wood, Joe

Woschke, Elmar

Woubishet, Taffese

Wouwer, Alain Vande

Woźniak, Łukasz

Wróbel, Karol

Wróblewski, Karol

Wróblewski, Łukasz

Wu, Bing

Wu, Kejun

$\mathrm{Wu}$, Panruo

Wu, Rome-Ming

Wu, Wei 
Wu, Xiangfa

$\mathrm{Wu}$, Xuejian

Wukovits, Walter

Wulandana, Rachmadian

Wurm, Florian

Wuttke, Manfred

Wyleciał, Tomasz

Wysocka, Izabela

Xavier, Joana C.

Xi, Guifa

$\mathrm{X}_{\mathrm{i}}$, Xuedong

Xia, Fangzhou

Xia, Shiwei

Xiang, Junting

Xiao, Boqi

Xiao, Dunhui

Xiao, Tiancun

Xiaoli Tan, Xiaoli

Xie, Haiyan

Xie, Hongchao

Xin, Liu

Xiong, Jia

$\mathrm{Xu}, \mathrm{Bin}$

$\mathrm{Xu}$, Chao

$\mathrm{Xu}$, Dikai

$\mathrm{Xu}$, Fei

$X u$, Jing

$\mathrm{Xu}$, Jinze

$\mathrm{Xu}$, Lang

$X u$, Pengfei

$\mathrm{Xu}$, Pengtao

$\mathrm{Xu}$, Rongguang

$\mathrm{Xu}$, Rongting (Tina)

$\mathrm{Xu}$, Shu

$X u, X i a$

$\mathrm{Xu}$, Xiaojie

$\mathrm{Xu}$, Xuesong

Yablonsky, Gregory

Yablonsky, Gregory S.

Yadav, Sakshi

Yadav, Sudesh

Yadavali, Sagar

Yaeno, Takashi

Yaici, Wahiba

Yakhvarov, Dmitry

Yakimovich, Artur

Yakobson, Boris A.

Yakush, Sergey E.

Yamada, Toru

Yamaki, Takehiro

Yamamoto, Makoto
Yan, Kai

Yan, Yonghua

Yang, Chih-Te

Yang, Guang

Yang, Hairui

Yang, Huaiwen

Yang, Huaiyu

Yang, MinoYang

Yang, Shaowei

Yang, Sheng

Yang, Shih Chun

Yang, Wendong

Yang, Ying

Yarlagadda, Prasad

Yasunaga, Takeshi

Yazdani, Maziar

Yazdani, Morteza

Ye, Liyun

Ye, Ruquan

Yeh, Ting-feng

Yehezkeli, Omer

Yeih, Wei-Chung

Yen, Benjamin

Yermán, Luis

Yilmaz, Muhittin

Yllmaz, Naz

Yiouta-Mitra, Paraskevi

Yong, Xin

Yoo, Hyun Ju

Yoo, Jihyung

Yoon, Dae-Ho

Yoon, Joon Yong

Yoon, Seongkyu

Yoshihara, Hiroshi

You, Byoung He

You, Shingchern

Yousef, Samy

Yousefian, Sajjad

Youssef, Tarek

Yu, Aimin

$\mathrm{Yu}$, Jiali

Yu, Kang

Yu, Manzhu

Yu, Wei

$\mathrm{Yu}$, Wenbin

$\mathrm{Yu}, \mathrm{Xi}$

$\mathrm{Yu}$, Zaiqun

Yuan, Hao

Yuan, Tao

Yuen, Anthony Chun Yin

Yunusa-Kaltungo, Akilu 
Yurchenko, Olga

Yurukcu, Mesut

Yvergnaux, Florent

Zabini, Federica

Zabkowski, Tomasz

Zablotsky, Dmitry

Zaborowska, Magdalena

Zaccone, Claudio

Zacharof, Myrto-Panagiota

Zadeh, Zahra Echresh

Zadravec, Manuela

Zaffora, Andrea

Zaidi, Syed Shan-E-Ali

Zaitsev, Alexander

Zajemska, Monika

Zakharov, Alex V.

Zakinyan, Arthur

Zamani, Nematollah

Zambon, Alfonso

Żamojć, Krzysztof

Zamostny, Petr

Zamparas, Miltiadis

Zanella, Mattia

Zanetti, Stefania

Zang, Guiyan

Zanjanijam, Ali Reza

Zanut, Alessandra

Zardin, Barbara

Zarzuelo Castañeda, Aránzazu

Zarzuelo, Carmen

Zavadlav, Sandra

Zavos, Anastasios

Zawada, Katarzyna

Zawala, Jan

Zawała, Jan

Zawieja, Iwona

Żbikowska, Anna

Zemek, Martin

Zendehboudi, Sohrab

Zeng, Jia

Zeng, Xiangze

Zgórka, Grażyna

Zgureva, Denitza

Zhai, Yao

Zhalniarovich, Yauheni

Zhan, Meng

Zhang, Bo

Zhang, Changyi

Zhang, Han

Zhang, Hongbo

Zhang, Hui
Zhang, Jiaxin

Zhang, Jinnan

Zhang, Jun

Zhang, Lifeng

Zhang, Minfang

Zhang, Qi

Zhang, Qichun

Zhang, Shiqiang

Zhang, Sui

Zhang, Tao

Zhang, Wei

Zhang, Weizhong

Zhang, Xuewei

Zhang, Yang

Zhang, Yi

Zhang, Youwen

Zhang, Yu

Zhang, Zhenhua

Zhang, Zhien

Zhang, Zhiyun

Zhao, Lianfeng

Zhao, Qiuying

Zhao, Shiquan

Zhao, Yadong

Zhao, Yao

Zhao, Yaqian

Zhao, Yongsheng

Zheng, Xiaorong

Zhou, Hui

Zhou, Jiajing

Zhou, Qixin

Zhou, Ran

Zhou, Shanshan

Zhou, Wenbin

Zhou, Xinyang

Zhou, Yiqun

Zhou, Yubin

Zhou, Zheyu

Zhu, Jiqing

Zhu, Xiaowei

Zhuo, Yueran

$\mathrm{Zi}$, Lin

Ziarno, Małgorzata

Zicmane, Inga

Zidek, Jan

Ziegenbalg, Dirk

Zieniuk, Bartłomiej

Zille, Andrea

Zimmerman, William

Zimoch-Korzycka, Anna

Zink, Béla 
Živković, Luka

Zivkovic, Vladimir

Žižková, Nikol

Zjavka, Ladislav

Złotek, Urszula

Zoładek, Maciej

Zolfaghari, Mehdi

Zolotukhin, Denis B.

Zotovic Stanisic, Ranko

Zoumpanioti, Maria

$\mathrm{Zu}$, Xingxing

Zubitur, Manoli M.
Zubowicz, Tomasz

Zubrik, Anton

Żubrowska-Sudoł, Monika

Zubrzycki, Jarosław

Zuccari, Fabrizio

Zueva, Svetlana B.

Zukowski, Witold

Zulli, Paul

Zuniga, Cristal

Zuntar, Irena

Zuorro, Antonio

Žurovec, David 